\title{
Moving Majorana bound states between distinct helical edges across a quantum point contact
}

\author{
Alessio Calzona $\odot^{1}$ and Björn Trauzettel ${ }^{1,2}$ \\ ${ }^{1}$ Institute of Theoretical Physics and Astrophysics, University of Würzburg, 97074 Würzburg, Germany \\ ${ }^{2}$ Würzburg-Dresden Cluster of Excellence ct.qmat, Germany
}

(Received 16 September 2019; published 31 December 2019)

\begin{abstract}
Majorana bound states are zero-energy excitations of topological superconductors that obey non-Abelian exchange statistics and are basic building blocks for topological quantum computation. To observe and exploit their extraordinary properties, we need to be able to properly manipulate them, for instance by braiding a couple of them in real space. We propose a setup based on the helical edges of two-dimensional topological insulators (2DTIs) that allows for a high degree of tunability by only controlling a handful of superconducting phases. In particular, our setup allows us to move the Majoranas along a single edge and to move them across two different edges coupled by a quantum point contact. Robustness against nonoptimal control of the phases is also discussed. This proposal constitutes an essential step forward toward realizing 2DTI-based architectures capable of performing braiding of Majoranas in a feasible way.
\end{abstract}

DOI: 10.1103/PhysRevResearch.1.033212

\section{INTRODUCTION}

Topological superconductors have been predicted to support Majorana bound states (MBSs), which are zero-energy midgap modes featuring non-Abelian braiding statistics. In addition to the interest in a fundamentally new excitation, the enormous potential of MBSs as building blocks for topological quantum computation has made their study one of the most active research fields in condensed-matter physics [1-5]. In this respect, the most advanced experimental platform to engineer MBSs is represented by semiconducting nanowires with strong spin-orbit coupling, proximitized by a conventional superconductor, in the presence of an external magnetic field [6-8]. This setup has been tested in several ground-breaking experiments, which provided strong evidence for the existence of MBSs by measuring zero-bias conductance peaks [9-15].

Several interesting alternative setups have been proposed, relying, for example, on magnetic adatoms [16-19], vortices in topological superconductors [20-22], quantum dots [23,24], or planar Josephson junctions [25-32]. In this respect, a promising system that hosts MBSs is based on the helical edge states of quantum spin Hall insulators (QSHIs) [33-36]. In this setup, MBSs emerge when parts of the edge are gapped out by superconducting and ferromagnetic barriers [37-43]. While being potentially more robust against multimode and disorder effects, the experimental quest for MBSs in QSHIbased devices has proven to be challenging. Nonetheless, superconductivity has been successfully proximity-induced into QSHIs, and experiments based on a helical Josephson junction have provided evidence for the formation of MBSs

Published by the American Physical Society under the terms of the Creative Commons Attribution 4.0 International license. Further distribution of this work must maintain attribution to the author(s) and the published article's title, journal citation, and DOI. by inspecting (missing) Shapiro steps [44]. Moreover, recent breakthroughs in the fabrication of QSHI-based devices have enabled us to couple different helical edges through a quantum point contact (QPC) [45].

Helical edge states are therefore likely to become a fertile playground to study the emergence of MBSs and their particular braiding properties, whose experimental observation is still lacking. To inspect the latter, indeed, systems with a high degree of tunability are required: in general, one has to deal with multiple couples of MBSs, to tune their couplings [46] and/or to vary their positions [47].

Seeking a realistic QSHI-based platform that provides the desired tunability represents the main task of this paper. The starting point is the well-known SFS architecture [37], where a single helical edge state is gapped by a finite ferromagnetic region $(\mathrm{F})$ that lies in between two semi-infinite superconducting barriers (S). In this system, a single couple of MBSs emerges and its hybridization can be tuned by acting on the superconducting phases [38,40]. Remarkably, we demonstrate that the insertion of an additional finite superconducting region greatly enhances the versatility of the system, allowing us to physically move the MBS along the edge. Such a displacement, which is again controlled exclusively by the superconducting phases, turns out to be independent of the degree of hybridization.

More importantly, the proposed SSFS architecture represents the fundamental building block of a multiedge setup, where one can move several zero-energy MBSs within the whole system (i.e., even between distinct edges). In this paper, we focus in particular on two edges of a 2DTI with SSFS geometry, locally coupled by a QPC. While being experimentally feasible, the proposed setup features great versatility when it comes to the manipulation of Majoranas. In particular, it allows us to move one zero-energy MBS from one edge to the other by exploiting electron tunneling at the QPC while the second MBS is kept fixed. Such a straightforward operation, performed by controlling only a handful of superconducting 


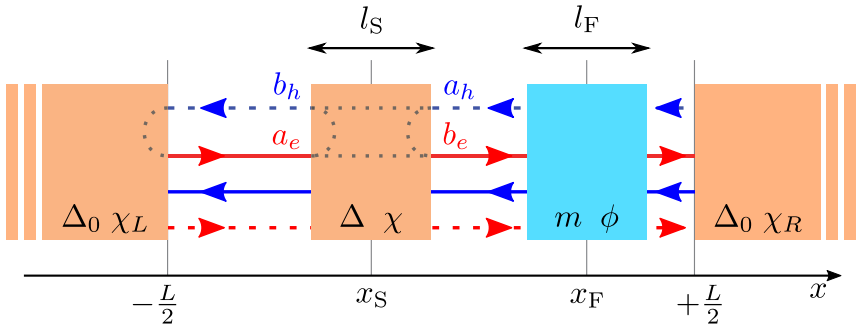

FIG. 1. The SSFS setup, a single helical edge gapped by superconductors (orange boxes) and a ferromagnet (blue box). Electrons (holes) are indicated by solid (dashed) lines, whose color refers to their spin: red (blue) for spin up (down). Some scattering processes, discussed in the main text, are depicted with gray dotted lines.

phases, clearly represents an essential step toward the physical braiding of Majoranas in 2DTI-based architectures.

\section{MOVING MAJORANAS ALONG A SINGLE EDGE}

At first, we focus on a single helical edge channel of a QSHI gapped by both superconducting and ferromagnetic regions, as shown in Fig. 1(a). The system Hamiltonian can be conveniently expressed as $H=\frac{1}{2} \int d x \Psi^{\dagger} \mathcal{H}_{\mathrm{BdG}} \Psi$ with the well-known Bogoliubov-de Gennes (BdG) Hamiltonian

$$
\mathcal{H}_{\mathrm{BdG}}(x)=p_{x} \tau_{3} \sigma_{3}+\vec{m}(x) \cdot \vec{\sigma}+\vec{\Delta}(x) \cdot \vec{\tau}-\mu(x) \tau_{3}
$$

and the Nambu spinor $\Psi=\left(\psi_{R \uparrow}, \psi_{L \downarrow}, \psi_{L \downarrow}^{\dagger},-\psi_{R \uparrow}^{\dagger}\right)^{T}$. The electron field operators $\psi_{r, s}$ annihilate a right- $(r=R)$ or left- $(r=L)$ moving particle with spin $s=\uparrow, \downarrow$ quantized along the $z$ axis. The Pauli matrices $\vec{\sigma}=\left(\sigma_{1}, \sigma_{2}, \sigma_{3}\right)[\vec{\tau}=$ $\left.\left(\tau_{1}, \tau_{2}, \tau_{3}\right)\right]$ act on spin (particle-hole) space, $p_{x}=-i \partial_{x}$ is the momentum operator, $\mu(x)$ is the chemical potential, and we have set both $\hbar=1$ and the Fermi velocity $v_{\mathrm{F}}=1$. The superconducting pairing and the Zeeman coupling read $\vec{\Delta}=(\Delta \cos \chi, \Delta \sin \chi, 0)^{T}$ and $\vec{m}=\left(m \cos \phi, m \sin \phi, m_{z}\right)^{T}$, respectively. As depicted in Fig. 1, the system we are interested in consists of three normal gapless regions with $m=\Delta=0$, which lie in between regions gapped by either a finite pairing potential $\Delta \neq 0(\mathrm{~S})$ or by a finite in-plane magnetization $m \neq 0(\mathrm{~F})$. The semi-infinite superconductors at the two ends $x= \pm L / 2$ lead to perfect Andreev reflections within the superconducting gap $\Delta_{0}$. For the sake of simplicity, in the following we will consider $\mu=m_{z}=0$ everywhere. The results we find, however, hold also in the presence of finite chemical potential and/or magnetization along the $z$ direction (see Appendix C). Moreover, we assume all the parameters to be uniform within each region.

It is well known that solutions of the $\mathrm{BdG}$ equation $\mathcal{H}_{\mathrm{BdG}} \varphi_{\epsilon}=\epsilon \varphi_{\epsilon}$ with energies $|\epsilon|<\Delta_{0}$ represent midgap bound states, described by the Nambu wave function $\varphi_{\epsilon}(x)$. The built-in particle-hole symmetry of the BdG Hamiltonian ensures that the bound states always come in pairs with opposite energies. Indeed, given an eigenstate $\varphi_{\epsilon}$, its chargeconjugated partner $\mathcal{C} \varphi_{\epsilon}$ is still an eigenstate of $\mathcal{H}_{\mathrm{BdG}}$ with opposite energy $-\epsilon$, where the operator $\mathcal{C}=\mathcal{K} \sigma_{2} \tau_{2}$, with $\mathcal{K}$ the complex conjugation [38]. As a remarkable consequence, whenever present, bound states at zero energy are always (at least) doubly degenerate and represent Majorana fermions.
Indeed, it is always possible to describe the two states in terms of two wave functions that are invariant under chargeconjugation, i.e., $\mathcal{C} \varphi_{0, j}=\varphi_{0, j}$ with $j=\alpha, \beta[38,48]$. In general, the degeneracy can be lifted by acting on the system parameters. Then, the two MBSs hybridize and acquire finite excitation energies $\pm \epsilon_{\text {Maj }}$.

In the following, we argue that the SSFS system allows us to selectively move the Majorana wave functions by controlling only two superconducting phases. Moreover, such manipulation can be performed without modifying $\epsilon_{\mathrm{Maj}}$, in particular while keeping the MBS at zero energy. To properly set the stage for our result, however, it is worthwhile to briefly review the simpler SFS geometry [37-40]. It can be seen as a limiting case of our setup for $l_{S} \rightarrow 0$. In this setup, the system hosts a couple of zero-energy MBSs for $\chi_{R}-\chi_{L}=\pi$. By acting on the superconducting phases, their energy splitting can be tuned up to a maximum value $\epsilon_{\text {Maj }} \propto e^{-m l_{\mathrm{F}}}$ reached when $\chi_{R}-\chi_{L}=0$. Unfortunately, the lack of additional knobs does not allow us to control the position of the Majorana wave functions, which are always localized on the two sides of the ferromagnetic region [38]. Such a limitation can be nicely overcome just by considering the presence of an additional superconductor with finite $l_{\mathrm{S}}>0$.

To find the midgap bound states of the SSFS system, depicted in Fig. 1, we employ scattering theory. The computations of the scattering matrices, lengthy but straightforward, are described in Appendixes A and B. For clarity we only present a specific example, which helps to understand the physics of our proposed architecture. In particular, we focus on the left semi-infinite superconductor and on the superconductor at $x=x_{\mathrm{s}}$, i.e., only on the left part of Fig. 1. Since there are no ferromagnets under this restricted perspective, right-moving electrons $(e)$ with spin-up can only be Andreevreflected into left-moving holes $(h)$ with spin down and vice versa. We can thus concentrate only on these two particle species and exploit particle-hole symmetry to gain information about the others. While the general results discussed in the following will be obtained by numerically analyzing the scattering problem for all subgap energies, here we are primarily interested in the zero-energy Majoranas and we therefore focus on the zero-energy limit. This allows us to show the relation between incoming and outgoing scattering amplitudes (depicted in Fig. 1),

$$
\left(\begin{array}{l}
b_{h} \\
b_{e}
\end{array}\right)=\left(\begin{array}{cc}
-i e^{i \chi} \tanh \left(\Delta l_{\mathrm{S}}\right) & \operatorname{sech}\left(\Delta l_{\mathrm{S}}\right) \\
\operatorname{sech}\left(\Delta l_{\mathrm{S}}\right) & -i e^{-i \chi} \tanh \left(\Delta l_{\mathrm{S}}\right)
\end{array}\right)\left(\begin{array}{l}
a_{e} \\
a_{h}
\end{array}\right),
$$

where $\Delta, l_{\mathrm{S}}$, and $\chi$ are the proximity-induced pairing amplitude, the length, and the phase of the finite superconducting region, respectively. The boundary with the semiinfinite superconductor implies perfect Andreev reflection with $a_{e}=-i e^{-i \chi_{L}} b_{h}$, where $\chi_{L}$ is the phase of the semiinfinite superconductor. The combined effect of the two superconductors leads, therefore, to perfect Andreev reflection, $b_{e}=-i e^{-i \chi_{\text {eff }}} a_{h}$, with an effective phase shift

$$
\chi_{\mathrm{eff}}\left(\chi_{L}, \chi, \Delta l_{\mathrm{S}}\right)=\chi-\arg \left[\frac{e^{i \chi}+e^{i \chi_{L}} \tanh \left(\Delta l_{\mathrm{S}}\right)}{e^{i \chi_{L}}+e^{i \chi} \tanh \left(\Delta l_{\mathrm{S}}\right)}\right] .
$$


Hence, we conclude that our SSFS system supports zeroenergy MBSs whenever the condition

$$
\chi_{R}=\chi_{\mathrm{eff}}\left(\chi_{L}, \chi, \Delta l_{\mathrm{S}}\right)-\pi
$$

is met. Moreover, the ratio between the scattering amplitudes on both sides of the finite superconductor reads

$$
\rho=\left|\frac{b_{e}}{a_{e}}\right|^{2}=\cosh \left(2 \Delta l_{\mathrm{S}}\right)+\sinh \left(2 \Delta l_{\mathrm{S}}\right) \cos \left(\chi_{L}-\chi\right) .
$$

As long as $\Delta l_{\mathrm{S}}$ is large enough, we can hence trap a zeroenergy mode either between the two superconductors (when $\left.\chi_{L}-\chi=\pi\right)$ or to the right of the finite one $\left(\chi_{L}-\chi=0\right)$. The ratio $\rho$ is plotted in Fig. 2(b) for $\Delta l_{\mathrm{S}}=2$.

Equations (3) and (5) nicely show the versatility of the SSFS geometry: the phase difference $\chi-\chi_{L}$ controls the position of one MBS along the edge while the third superconducting phase $\chi_{R}$ can be used to independently tune the Majorana hybridization. This result is confirmed by the exact numerical study of the SSFS architecture (for all subgap energies). In the specific geometry that we consider, the lengths of the middle superconductor and the ferromagnet equal twice the superconducting coherence length $\xi=\Delta_{0}^{-1}$, i.e., $l_{\mathrm{S}}=l_{\mathrm{F}}=2 \xi$. The total length of the edge is $L=7 \xi$ and the middle superconductor and ferromagnet are located at $x_{\mathrm{S}}=$ $-x_{\mathrm{F}}=-1.5 \xi$, respectively. The hybridization between the two Majoranas is studied in Fig. 2(a), where we plotted $\epsilon_{\text {Maj }}$ as a function of the phase differences $\chi-\chi_{L}$ and $\chi_{R}-\chi_{L}$. We considered $m=\Delta=\Delta_{0}$, while the magnetization angle $\phi$ is not relevant. The red regions indicate a large hybridization, whose actual value exponentially depends on the strength $\left(m l_{\mathrm{F}}\right)$ of the ferromagnetic region, as in the standard SFS geometry. By contrast, the white-dashed line highlights points where $\chi_{R}$ satisfies the zero-energy condition in Eq. (4) and the two Majoranas are thus completely decoupled.

By changing the phase difference $\chi-\chi_{L}$ and tuning the third phase $\chi_{R}$ according to Eq. (4), it is possible to change the localization of the zero-energy Majoranas, without hybridizing them. In Fig. 2(b) we plot the ratio $\rho$ on a logarithmic scale, recalling that $\rho>1(\rho<1)$ corresponds to a zeroenergy Majorana wave function mainly localized to the right (left) of the middle superconductor. In Fig. 2(c) we plot the full subgap spectrum, which confirms the presence of the zero-energy Majoranas (black dashed line) together with other midgap Andreev bound states (ABSs) shown in gray. The spectrum is even in the phase difference $\chi-\chi_{L}$ and symmetric for $\epsilon \rightarrow-\epsilon$. We observe that, at $\chi-\chi_{L}=\pi$, the energy gap between the zero-energy Majoranas and the first ABS is small and it turns out to be exponentially suppressed in the strength $\Delta l_{\mathrm{S}}$ of the middle superconductor [49]. As a result, there is a tradeoff between the possibility to properly localize a Majorana on either side of the central superconductor [which requires a large $\Delta l_{S}$ according to Eq. (5)] and having a large energy gap between the zero-energy Majoranas and the other midgap ABS (which requires a small $\Delta l_{S}$ ). The numerical results in Fig. 2 show that $\Delta l_{S}=2$ is a good compromise.

To better visualize and discuss the localization of the zero-energy MBSs, we computed their wave functions by using scattering theory (see Appendix B) and we plot them in Fig. 2(d). In particular, we focus on three parameter

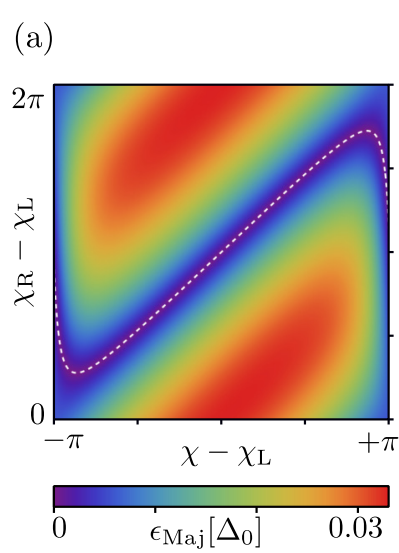

(b)
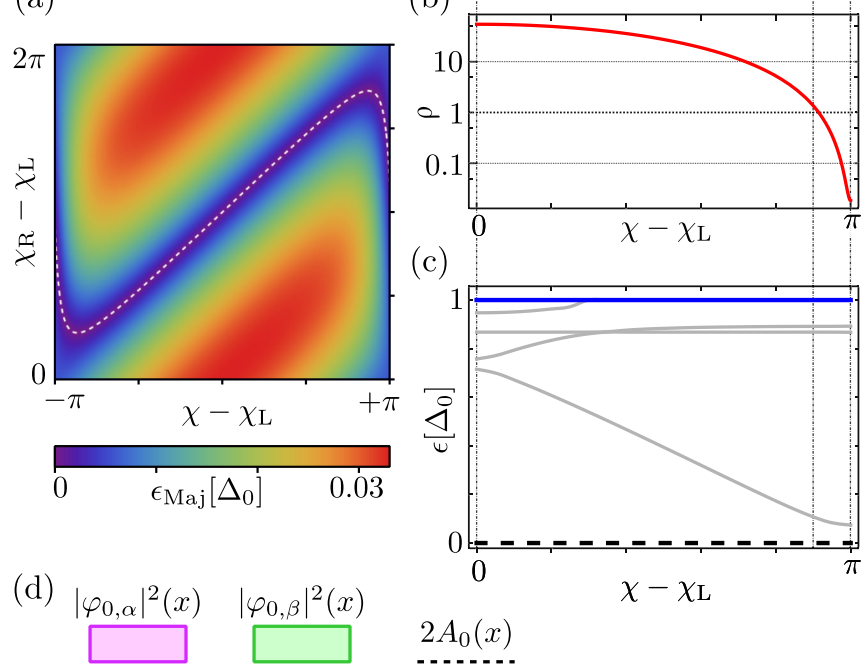

(c)

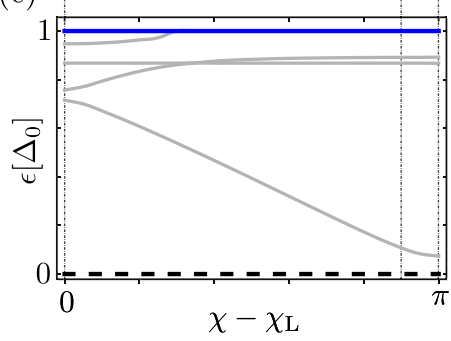

$2 A_{0}(x)$
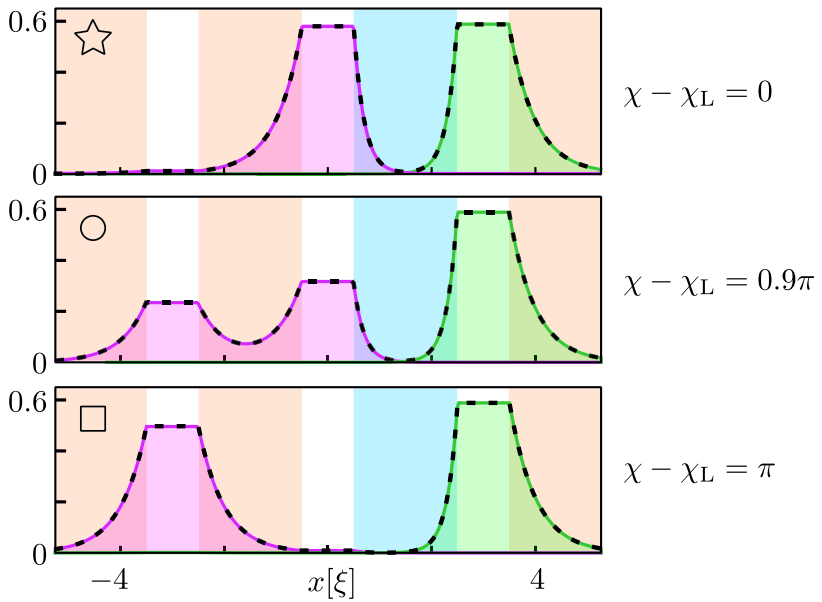

FIG. 2. (a) Energy splitting $\epsilon_{\text {Maj }}$ of the two Majoranas as a function of the superconducting phase differences, with $\Delta=m=$ $\Delta_{0}$. (b) Logarithmic plot of the ratio $\rho$ as a function of $\chi-\chi_{L}$, describing the localization of a zero-energy Majorana to the right $(\rho>1)$ or to the left $(\rho<1)$ of the central finite superconductor. (c) Spectrum of the system as a function of $\chi-\chi_{L}$, with $\chi_{R}$ satisfying the zero-energy condition of Eq. (4). The zero-energy Majoranas are depicted with the black-dashed line. Other ABSs are plotted with gray lines. The superconducting gap $\Delta_{0}$ is marked in blue. We consider $m=2.5 \Delta=2.5 \Delta_{0}$. (d) Zero-energy Majorana wave functions $\left|\varphi_{0, j}(x)\right|^{2}(x)$ along the edge, for different values of the phase difference $\chi-\chi_{L}=0$ (star), $0.9 \pi$ (circle), $\pi$ (square). The left MBS (purple) moves along the edge while the other one (green) remains fixed. The dotted black lines shows twice the zero-energy LDOS $2 A_{0}(x)$. We choose again $m=2.5 \Delta=2.5 \Delta_{0}$. Wave functions and LDOS are plotted in units $\xi^{-1}$. All the plots are computed for the following geometry: $l_{\mathrm{S}}=l_{\mathrm{F}}=\xi=\Delta_{0}^{-1}, L=7 \xi, x_{\mathrm{F}}=-x_{\mathrm{S}}=1.5 \xi$.

configurations that support zero-energy MBSs: $\chi-\chi_{L}=0$ (star), $\chi-\chi_{L}=0.9$ (circle), and $\chi-\chi_{L}=1$ (square) while always keeping $\chi_{\text {eff }}-\chi_{R}=\pi$. As a function of the phase difference $\chi-\chi_{L}$, the wave function $\left|\varphi_{0, \alpha}(x)\right|^{2}$ of the left Majorana (purple) moves across the finite superconductors, while the other one $\left|\varphi_{0, \beta}(x)\right|^{2}$ (green) is fixed to the right of the ferromagnetic region. Such a behavior directly affects the zero-temperature local density of states (LDOS) of the system 


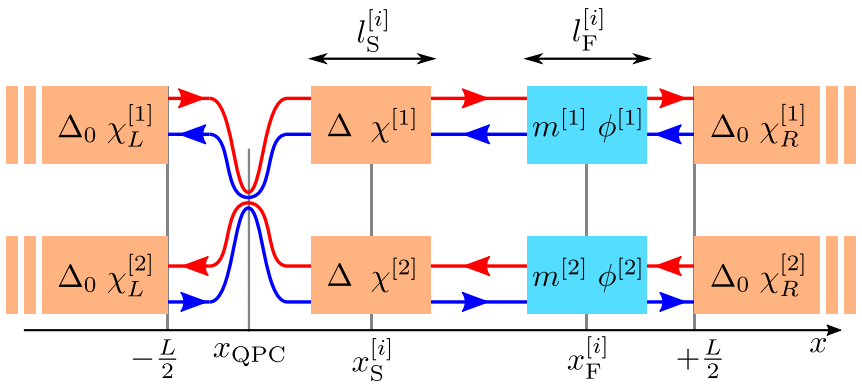

FIG. 3. Two helical edges with SSFS geometry, coupled by a QPC at $x=x_{\mathrm{QPC}}$. Orange (blue) boxes indicate superconducting (ferromagnetic) gapped regions. Red (blue) lines refers to electron channels with spin up (down).

(see Appendix E),

$$
\mathcal{A}(\omega, x)=\sum_{\epsilon} A_{\epsilon}(x) \delta(\omega-\epsilon),
$$

where the sum is taken over all the bound-state energies. Its zero-energy $A_{0}(x)$ component features indeed two peaks centered over the MBS and which therefore move accordingly, as shown in Fig. 2(d) with black dotted lines. We note in passing that our system can be seen as an example of an Andreev molecule [50], where the bound states of each gapless region hybridize with those of the neighbor gapless regions.

\section{MOVING MAJORANAS BETWEEN DIFFERENT EDGES}

The full manipulation of MBSs offered by the SSFS architecture represents itself an important achievement. However, a single couple of Majoranas constrained on a one-dimensional edge with open boundaries is not enough to detect and exploit their non-Abelian properties. It is thus necessary to consider systems consisting of several couples of MBSs, living on different helical edges, which have to be coupled to each other. In this respect, an intriguing and feasible possibility is represented by QPCs, which allow electron tunneling between the edges [45]. Remarkably, we prove that QPCs can be used for interedge MBS manipulation. Electron tunneling is indeed sensitive to the LDOS, which we just demonstrate to be controllable by moving the MBS along each edge within an SSFS architecture. As a result, the interedge coupling provided by the QPC can be effectively and efficiently tuned just by acting on a handful of superconducting phases. We will focus, in particular, on a simple double-edge configuration, which might be realized in current QPC systems [45].

The system we are considering is shown in Fig. 3 and consists of two SSFS edges, coupled by a QPC located between the superconductors at $x=x_{\mathrm{QPC}}$. As described in detail in Appendix D, its Hamiltonian reads [51] $\left(\vartheta_{R / L}= \pm 1\right.$, operators are evaluated at $x=x_{\mathrm{QPC}}$ )

$$
H_{t}=2 \lambda_{\mathrm{sp}} \sum_{\sigma=\uparrow, \downarrow} \psi_{R \sigma}^{\dagger} \psi_{L \sigma}+2 \lambda_{\mathrm{sf}} \sum_{r=R, L} \vartheta_{r} \psi_{r_{\uparrow}}^{\dagger} \psi_{r_{\downarrow}}+\text { H.c. }
$$

It models spin-preserving $\left(\lambda_{\mathrm{sp}}\right)$ and spin-flipping $\left(\lambda_{\mathrm{sp}}\right)$ tunneling between the channels of the upper edge $\left(R_{\uparrow}\right.$ and $\left.L_{\downarrow}\right)$ and the ones of the lower edge $\left(R_{\downarrow}\right.$ and $\left.L_{\uparrow}\right)$. Starting from
TABLE I. Quantitative description of the protocol that moves one zero-energy Majorana across the QPC.

\begin{tabular}{ccccc}
\hline \hline Edge & $\chi_{L}^{[j]}$ & $\chi^{[j]}$ & $\phi^{[j]}$ & $\chi_{R}^{[j]}$ \\
\hline$j=1$ & 0 & $r(y)$ & $\phi$ & $\pi+\chi_{\mathrm{eff}}^{[1]}(y)+f(y)$ \\
$j=2$ & $\pi$ & $r(y)+\pi$ & $\phi+\pi$ & $y\left[\pi+\chi_{\mathrm{eff}}^{[2]}(y)\right]+y 0.05 \pi+\pi$ \\
\hline \hline
\end{tabular}

the equation of motion, it is possible to derive the $8 \times 8$ scattering matrix $\mathcal{S}_{\mathrm{QPC}}$ associated with the QPC [51] and use the machinery developed in the previous section in order to study the bound states of the whole double-edge system. We mention in passing that the entire setup can also be viewed as a realization of a multiterminal Josephson junction [52,53]. In this section, we will specifically consider the following geometry (see Fig. 3 for notation):

$$
\begin{aligned}
3 l_{\mathrm{S}}^{[1,2]} & =2 \xi, & l_{\mathrm{F}}^{[1]} & =3 \xi, & & l_{\mathrm{F}}^{[2]}=2.4 \xi, \\
x_{\mathrm{S}}^{[1,2]} & =-1.5 \xi, & x_{\mathrm{F}}^{[1]} & =1.5 \xi, & & x_{\mathrm{F}}^{[2]}=1.8 \xi, \\
L & =7 \xi, & x_{\mathrm{QPC}} & =-3 \xi . & &
\end{aligned}
$$

We are now able to describe a protocol that enables moving one zero-energy MBS from the upper edge to the lower one. In particular, we start from a configuration that hosts two zeroenergy MBSs on the upper edge [see Fig. 4, top panel (star)], and we end with one zero-energy Majorana localized on each edge [see Fig. 4, bottom panel (cross)]. Before presenting a quantitative (and numerical) description of the protocol, it is useful to qualitatively show how the initial and final configurations can be achieved by exploiting the QPC and the SSFS architecture. To this end, we observe that when the whole system is tuned such that the LDOS (almost) vanishes in the QPC region, the two edges are effectively decoupled. We can then fully hybridize the MBS on the lower edge ( $j=$ 2), i.e., $\chi_{R}^{[j=2]} \simeq \chi_{\text {eff }}\left(\chi_{L}^{[2]}, \chi^{[2]}\right)$, while keeping the Majoranas on the upper edge $(j=1)$ at zero energy, i.e., $\chi_{R}^{[1]} \simeq \pi+$ $\chi_{\text {eff }}\left(\chi_{L}^{[1]}, \chi^{[1]}\right)$. This allows us to realize the initial configuration. As for the final one, we tune the system such that the two edges would host two couples of zero-energy MBSs localized close to the four semi-infinite superconductors, i.e., $\chi^{[j]}-\chi_{L}^{[j]} \simeq \pi$. In such a configuration, the QPC effectively couples and hybridizes the two left Majoranas, leaving at zero energy only the ones to the right of the ferromagnets.

We quantitatively design the protocol by interpolating between these initial and final configurations. The superconducting phases are tuned, according to Table I, as a function of a single parameter $y$ that ramps from 0 to 1 . The fixed $\pi$ phase difference between the two edges is essential in order for the QPC to effectively couple the MBS (see Appendix D). The function $r(y)$ reads

$$
r(y)=0.95 \pi \begin{cases}{\left[1-(2 y-1)^{4}\right],} & 0 \leqslant y<0.5, \\ 1, & 0.5 \leqslant y \leqslant 1,\end{cases}
$$

while the continuous function $f(y)$ provides a tiny correction $0 \leqslant f(y) \lesssim 0.15 \pi$ to ensure that the two MBSs are indeed exactly at zero energy (see Appendix F). The numerical computation of the bound states as a function of $y$ is performed by considering the QPC tunneling amplitudes $\lambda_{\mathrm{sp}}=0.1$ and 


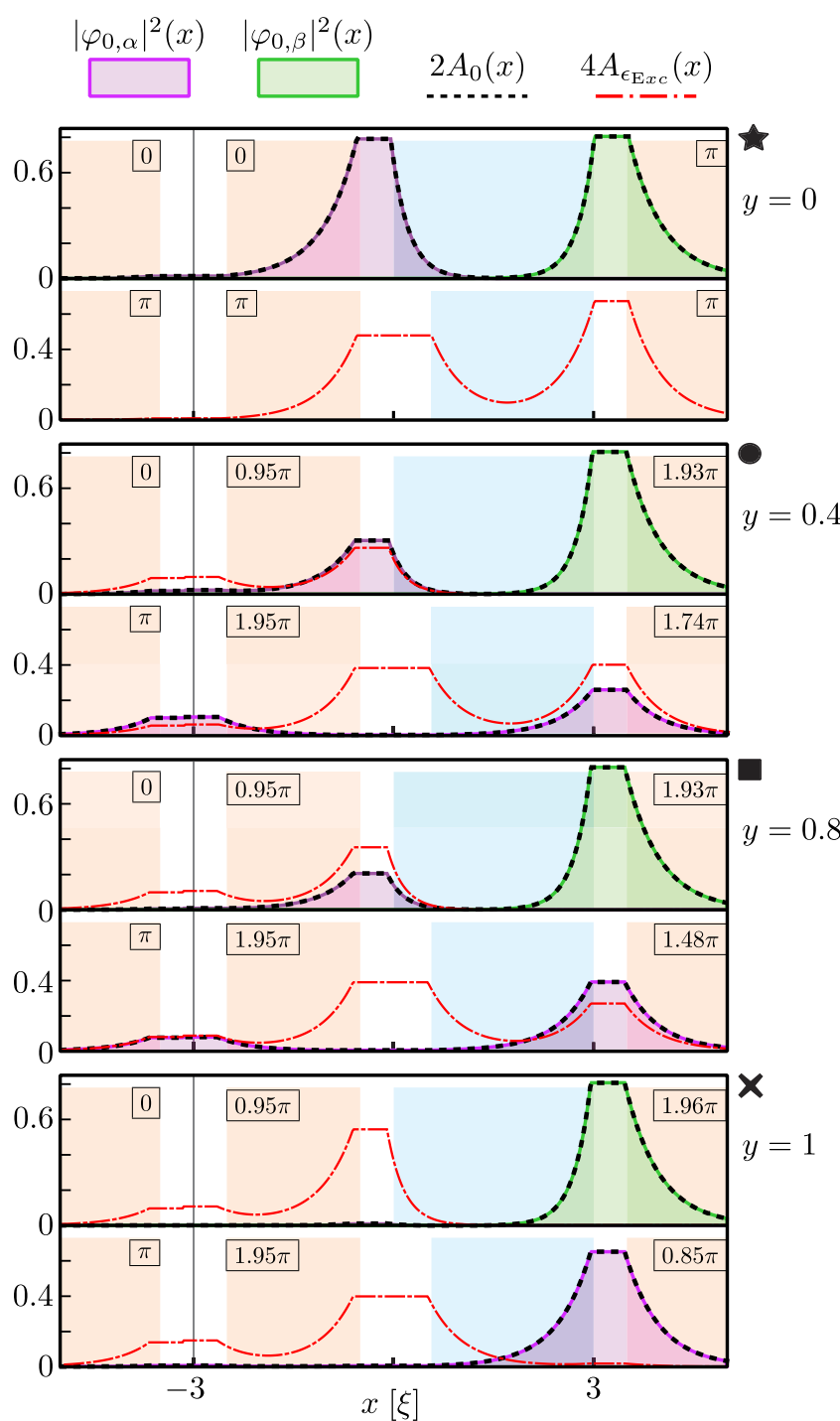

FIG. 4. Zero-energy Majorana wave functions $\left|\varphi_{0, \alpha}(x)\right|^{2}$ (green) and $\left|\varphi_{0, \beta}(x)\right|^{2}$ (purple) on the two edges. As $y$ ramps from 0 to 1 , the purple Majorana moves from the upper edge to the lower one while the green one remains fixed on the upper one. The black dotted lines show (twice) the zero-energy LDOS, i.e., $2 A_{0}(x)$. The thinner dashdotted red lines show (four times) the LDOS associated to the first excited state, i.e., $4 A_{\epsilon_{\mathrm{Exc}}}(x)$ with $\epsilon_{\mathrm{Exc}}>0$. In the little black boxes we report the superconducting phase of each superconductor, assuming $\chi_{L}^{[1]}=0$. We choose $m^{[1]}=2 m^{[2]}, \Delta=m^{[2]}=\Delta_{0}$. Wave functions and LDOS are plotted in units $\xi^{-1}$.

$\lambda_{\mathrm{sf}}=0$. The presence of spin-flipping processes does not qualitatively affect the results. We stress that the specific choice of $r(y)$, as well as the presence of a tiny correction $y 0.05 \pi$ in $\chi_{R}^{[2]}$, only aims at optimizing some features of the protocol but does not modify the qualitative description given above. Finally, as for the single-edge case, the magnetic angle $\phi$ is not relevant.

Figure 4 shows how the Majorana wave functions are manipulated during the protocol, and it represents one of the main results of our work. In particular, we plot the zero-energy MBS wave functions $\left|\varphi_{0, j}(x)\right|^{2}(j=\alpha, \beta)$ for four different values of $y=0,0.4,0.8,1$ (star, circle, square, and cross, respectively). The purple Majorana clearly moves from the upper edge to the lower one, while the green one sticks to the gapless region to the right of the upper ferromagnet. As discussed for the single-edge system, the motion of the Majoranas directly affects the LDOS at zero energy $A_{0}(x)$ (dotted black lines): one of its two peaks indeed moves from one edge to the other one. In the initial configuration $y=0$ (star), when the two edges are almost completely decoupled, it is clear that the system hosts also a couple of midgap bound states at finite energies $\pm \epsilon_{\mathrm{Exc}}$, resulting from the full hybridization of the MBS on the lower edge. They give rise to a nonvanishing spectral weight in the LDOS at finite energy $A_{\epsilon_{\mathrm{Exc}}}(x)$, which is plotted with dash-dotted red lines. These midgap states evolve as $y$ is ramped from 0 to 1 and eventually localize close to the QPC region, as expected from the qualitative description of the final configuration given above. The LDOS can be experimentally probed by performing tunneling spectroscopy [54], e.g., by exploiting an additional QPC near pinch-off.

While the two decoupled Majoranas remain at zero energy throughout the protocol, it is important to discuss the behavior of other midgap states with finite energy. To this end, in Fig. 5(b) we plot the subgap spectrum as a function of the parameter $y$. Right above the zero-energy Majoranas (blackdashed line), the red dash-dotted line represents the first excited midgap state with energy $\epsilon_{\mathrm{Exc}}(y)$, whose associated LDOS $A_{\epsilon_{\mathrm{Exc}}}(x)$ is plotted in Fig. 4 (with a red dash-dotted line). Importantly, there is always a finite-energy gap between this state and the zero-energy Majoranas. In particular, with the protocol described in Table I and with the interpolating function $r(y)$ in Eq. (8), one has $\epsilon_{\operatorname{Exc}}(y) \gtrsim 0.04 \Delta_{0}$. Other ABSs with higher energy are plotted with gray lines.

To implement the protocol, it is necessary to control the phase differences across the helical Josephson junctions. A possibility is to tune magnetic fluxes $[47,54-56]$ using, for example, the architecture suggested in Fig. 5(d). In this case, one would have to vary $\left[\Phi_{0}=h /(2 e)\right.$ is the flux quantum]

$$
\begin{gathered}
\Phi_{B}(y)=-\Phi_{0} \frac{r(y)}{2 \pi}, \\
\Phi_{C}(y)=\Phi_{0} \frac{r(y)}{2 \pi}, \\
\Phi_{D}(y)=-\Phi_{0} \frac{\pi+\chi_{\mathrm{eff}}^{[1]}(y)+f(y)-r(y)}{2 \pi}, \\
\Phi_{E}(y)=\Phi_{0} \frac{y\left[\pi+\chi_{\mathrm{eff}}^{[2]}(y)\right]+y 0.05 \pi-r(y)}{2 \pi},
\end{gathered}
$$

while

$$
\Phi_{A}=\frac{\Phi_{0}}{2}
$$

is fixed. The dependence of these magnetic fluxes on the parameter $y$ is shown in Fig. 5(a).

It is important to stress that in our setup, the degeneracy of the two zero-energy Majoranas is not topologically protected since its splitting $\epsilon_{\text {Maj }}$ is not exponentially small in the deviations of superconducting phases from their optimal values. It is therefore interesting to discuss the robustness of the proposed protocol with respect to a finite accuracy of the phase control. To this end, we add an uncorrelated Gaussian noise with standard deviation $\pi / 20$ to each phase indepen- 

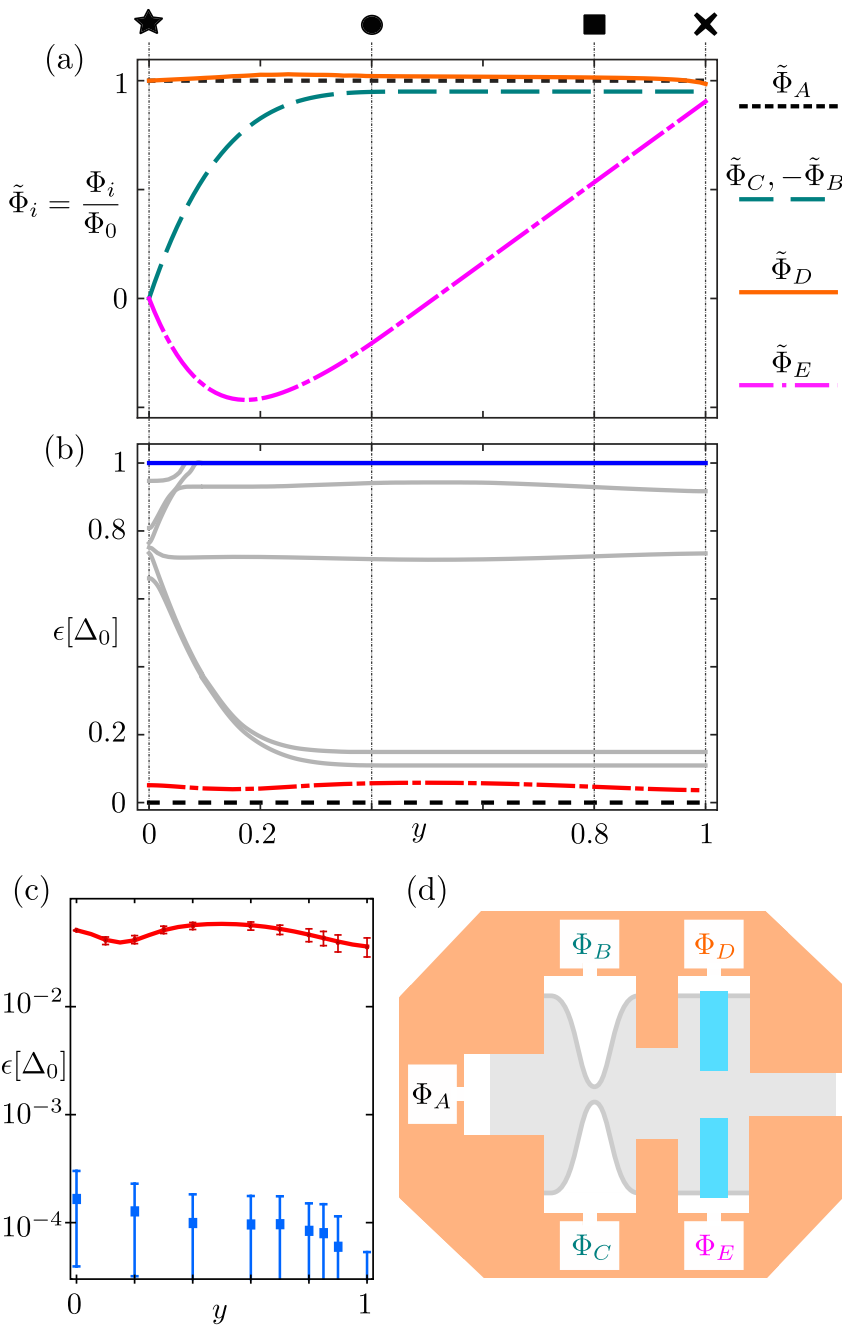

(d)

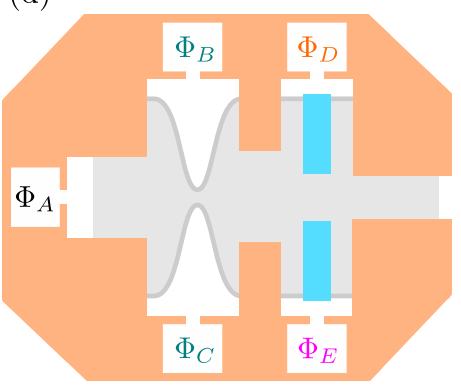

FIG. 5. (a) Dependence of the magnetic fluxes [see panel (d)] on the parameter $y$ throughout the protocol. (b) Subgap spectrum of the system as a function of $y$. One can identify the zero-energy Majoranas (black dashed line), the first excited state with energy $\epsilon_{\text {Exc }}(y)$ (red dash-dotted line), other midgap ABSs (gray lines), and the superconducting gap $\Delta_{0}$ (blue line). The spectrum is symmetric with respect to $\epsilon \rightarrow-\epsilon$. The black markers show the specific values of $y$ considered in Fig. 4. (c) Logarithmic plot of the low-energy part of the spectrum in the presence of finite accuracy in the phase control. Blue and red markers show $\epsilon_{\mathrm{Maj}}(y)$ and $\epsilon_{\mathrm{Exc}}(y)$, respectively. The error bars represent the standard deviations of every point, while the red line shows $\epsilon_{\mathrm{Exc}}(y)$ as a reference. (d) A possible scheme to tune the phase differences across the helical Josephson junction by using magnetic fluxes $\Phi_{i}$. Different colors refer to superconductors (orange), ferromagnets (light blue), and 2DTI (gray). For all the plots, we choose $m^{[1]}=2 m^{[2]}, \Delta=m^{[2]}=\Delta_{0}$.

dently and then average over many realizations for each given $y$. This finite accuracy affects the energies of all midgap states, and we focus in particular on the zero-energy Majoranas $\left(\epsilon_{\text {Maj }}\right)$ and on the first excited state $\left[\epsilon_{\text {Exc }}(y)\right]$. As shown in Fig. 5(c), a nonoptimal control of the superconducting phases leads to a degeneracy splitting $\tilde{\epsilon}_{\text {Maj }} \sim 10^{-4} \Delta_{0}$ (blue markers) and to a modification of $\epsilon_{\text {Exc }}(y) \rightarrow \tilde{\epsilon}_{\text {Exc }}(y)$ (red markers). The error bars show the standard deviation associated with each point while the red line shows $\epsilon_{\mathrm{Exc}}(y)$ as a reference.
Importantly, we observe that even with limited accuracy in the phase control (with errors of the order of $\pi / 20$ ), there are still more than two orders of magnitude between the two almost-zero-energy Majoranas and the other midgap states. As argued in the following paragraphs, this is still compatible with a successful implementation of the protocol.

\section{DISCUSSION AND CONCLUSIONS}

Our proposal, although challenging, is experimentally feasible. Indeed, it has been shown that it is possible to proximitize the helical edges by using $\mathrm{Al}$ superconducting contacts [44,57,58]. The superconducting coherence length is $\xi \sim$ $0.6-2 \mu \mathrm{m}$ while the induced superconducting gap is $\Delta \sim$ $90 \mu \mathrm{eV}$. Moreover, the ballistic mean free path on the helical edges is estimated to be $l_{\mathrm{b}} \sim 2.4 \mu \mathrm{m}$. Regarding quantum point contacts, the physical size of the first-realized QPC in $\mathrm{HgTe} 2 \mathrm{DTI}$ is around $l_{\mathrm{QPC}} \sim 0.5 \mu \mathrm{m}$ [45]. It is therefore reasonable to assume our helical gapless regions, whose lengths are of the order of $\xi$, to be wide enough to accommodate a QPC but short enough to assume ballistic transport. As for the ferromagnetic regions, we assumed $m \sim \Delta_{0}$, which corresponds to $m / \mu_{0} \sim 1 \mathrm{~T}$, where $\mu_{0}$ is the Bohr magneton.

The energy gap between the Majoranas and the first excited midgap states is of the order of $\epsilon_{\mathrm{Exc}} \sim 4 \mu \mathrm{V}$, which corresponds to a temperature scale $T_{\mathrm{Exc}}=\epsilon_{\mathrm{Exc}} / k_{\mathrm{B}} \sim 5 \times 10^{-2} \mathrm{~K}$ and to a timescale $t_{\mathrm{Exc}}=h / \epsilon_{\mathrm{Exc}} \sim 1 \mathrm{~ns}$. As long as the protocol is performed over a time span $t_{p}$ much greater than $t_{\mathrm{Exc}}$, we can safely assume an adiabatic evolution of the system. In general, upper bounds to the total moving time $t_{p}$ exist as well. One might be represented by the quasiparticle poisoning time $t_{\mathrm{qp}}$, which depends strongly on the superconducting system $[59,60]$, but should in any case exceed hundreds of nanoseconds [61]. Another timescale $t_{\mathrm{GS}}$ arises when the ground-state degeneracy is split, for example because of a finite accuracy in tuning the superconducting phases. In this case, for an accuracy up to $\pi / 20$, one has $t_{\mathrm{GS}}=h / \tilde{\epsilon}_{\mathrm{Maj}} \sim 410^{2}$ ns. If $t_{p}$ exceeds this timescale, the difference in the dynamical phases gained by the two lowest-energy states is not negligible anymore. In general, however, it is possible to operate at an intermediate regime $t_{\mathrm{Exc}} \ll t_{p} \ll t_{\mathrm{GS}}, t_{\mathrm{qp}}$.

The proposed SSFS geometry allows us to achieve a promising tunability of a single couple of MBSs on the helical edge of a 2DTI. In particular, we can independently move the Majoranas along the edge and tune their hybridization. While already interesting on its own, this architecture develops its full potential when implemented on multiple edges, coupled via a QPC. The interplay between interedge electron tunneling and intraedge Majorana manipulation results in the possibility of fully controlling the position of a single MBS over the whole system, just by tuning a handful of superconducting phases. In particular, the present proposal deals with a simple double-edge configuration, which might be realized in current QPC systems on a 2DTI. We invent a protocol that allows us to move one MBS from the upper to the lower edge while leaving the other MBS untouched. This change in the position of a MBS, controlled by tuning four superconducting phase differences, directly affects the zero-energy LDOS of the system.

The combination of the SSFS geometry with the QPC dramatically enhances the capabilities of 2DTI systems in 
manipulating MBSs. Despite the lack of topological protection, our proposal paves the way for a challenging but feasible implementation of braiding schemes that can reveal the nonAbelian nature of Majoranas.

\section{ACKNOWLEDGMENTS}

We would like to thank M. Carrega, C. Fleckenstein, F. Keidel, M. Stehno, and S. Zhang for fruitful discussion. We acknowledge support by the Würzburg-Dresden Cluster of Excellence ct.qmat (EXC2147, project-id 39085490).

\section{APPENDIX A: BDG HAMILTONIAN}

We first analyze the Hamiltonian of a single helical edge in the presence of superconducting and ferromagnetic regions. The latter can be conveniently expressed as

$$
H=\frac{1}{2} \int d x \Psi^{\dagger} \mathcal{H}_{\mathrm{BdG}} \Psi,
$$

with the Nambu spinor

$$
\Psi=\left(\psi_{\uparrow}, \psi_{\downarrow}, \psi_{\downarrow}^{\dagger},-\psi_{\uparrow}^{\dagger}\right)^{T}
$$

and the Bogoliubov-de Gennes Hamiltonian

$$
\mathcal{H}_{\mathrm{BdG}}(x)= \pm p_{x} \tau_{3} \sigma_{3}+\vec{m}(x) \cdot \vec{\sigma}+\vec{\Delta}(x) \cdot \vec{\tau}-\mu(x) \tau_{3} .
$$

The sign in front of the momentum operator selects the helicity of the edge: a plus (minus) sign corresponds to rightmoving electrons with spin up (down) and vice versa. The Pauli matrices $\vec{\sigma}=\left(\sigma_{1}, \sigma_{2}, \sigma_{3}\right)\left[\vec{\tau}=\left(\tau_{1}, \tau_{2}, \tau_{3}\right)\right]$ act on spin (particle-hole) space, $p_{x}=-i \partial_{x}$ is the momentum operator, $\mu(x)$ is the chemical potential, and we have set both $\hbar=$ 1 and the Fermi velocity $v_{\mathrm{F}}=1$. The superconducting and ferromagnetic regions are described by nonvanishing,

$$
\begin{aligned}
\vec{\Delta} & =(\Delta \cos \chi, \Delta \sin \chi, 0)^{T}, \\
\vec{m} & =\left(m \cos \phi, m \sin \phi, m_{z}\right)^{T},
\end{aligned}
$$

respectively. The Hamiltonian can be diagonalized as

$$
H=\sum_{\epsilon \geqslant 0} \sum_{j} \epsilon \gamma_{\epsilon, j}^{\dagger} \gamma_{\epsilon, j},
$$

where the operators $\gamma_{\epsilon_{n}, j}^{\dagger}$ and $\gamma_{\epsilon, j}$ create and annihilate a fermionic quasiparticle with energy $\epsilon$, respectively [38]. The index $j$ takes into account possible degeneracies. The energy of the ground state(s) has been set to zero. The diagonalized form (A6) is achieved by exploiting the ansatz on the Nambu spinor,

$$
\Psi(x)=\sum_{\epsilon \geqslant 0} \sum_{j} \varphi_{\epsilon, j}(x) \gamma_{\epsilon, j}+\left[\mathcal{C} \varphi_{\epsilon, j}\right](x) \gamma_{\epsilon, j}^{\dagger},
$$

where

$$
\varphi_{\epsilon, j}=\left(u_{\epsilon, j, \uparrow}, u_{\epsilon, j, \downarrow}, v_{\epsilon, j, \downarrow}, v_{\epsilon, j, \uparrow}\right)^{T}
$$

is a solution of the $\mathrm{BdG}$ equation,

$$
\mathcal{H}_{\mathrm{BdG}} \varphi_{\epsilon, j}=\epsilon \varphi_{\epsilon, j},
$$

and $\mathcal{C}=\mathcal{K} \tau_{2} \sigma_{2}$, with $\mathcal{K}$ the complex conjugation, is the charge-conjugation operator [38]. The BdG Hamiltonian (A3) features a built-in particle-hole symmetry,

$$
\mathcal{C} \mathcal{H}_{\mathrm{BdG}} \mathcal{C}^{-1}=-\mathcal{H}_{\mathrm{BdG}}
$$

which implies

$$
\mathcal{H}_{\mathrm{BdG}} \varphi_{\epsilon, j}=\epsilon \varphi_{\epsilon, j} \Rightarrow \mathcal{H}_{\mathrm{BdG}}\left(\mathcal{C} \varphi_{\epsilon, j}\right)=-\epsilon\left(\mathcal{C} \varphi_{\epsilon, j}\right) .
$$

When degeneracies are not present, one can suppress the index $j$ and exploit the identity (up to a global phase)

$$
\left(\begin{array}{c}
u_{\uparrow}(\epsilon) \\
u_{\downarrow}(\epsilon) \\
v_{\downarrow}(\epsilon) \\
v_{\uparrow}(\epsilon)
\end{array}\right)=\left(\begin{array}{c}
-v_{\uparrow}(-\epsilon)^{*} \\
v_{\downarrow}(-\epsilon)^{*} \\
u_{\downarrow}(-\epsilon)^{*} \\
-u_{\uparrow}(-\epsilon)^{*}
\end{array}\right)
$$

\section{Bogoliubov equations}

Solving the Bogoliubov-de Gennes equation allows us to determine the expressions for the wave-function components $u_{\uparrow, \downarrow}(\epsilon, x)$ and $v_{\uparrow, \downarrow}(\epsilon, x)$ of the bound states. To this end, we transform the equation

$$
\pm\left(-i \partial_{x}\right)\left(\begin{array}{cccc}
1 & 0 & 0 & 0 \\
0 & -1 & 0 & 0 \\
0 & 0 & -1 & 0 \\
0 & 0 & 0 & 1
\end{array}\right)\left(\begin{array}{l}
u_{\uparrow} \\
u_{\downarrow} \\
v_{\downarrow} \\
v_{\uparrow}
\end{array}\right)+\left(\begin{array}{cccc}
-\mu-\epsilon+m_{z} & m e^{-i \phi} & \Delta e^{-i \chi} & 0 \\
m e^{i \phi} & -\mu-\epsilon-m_{z} & 0 & \Delta e^{-i \chi} \\
\Delta e^{i \chi} & 0 & \mu-\epsilon+m_{z} & m e^{-i \phi} \\
0 & \Delta e^{i \chi} & m e^{i \phi} & \mu-\epsilon-m_{z}
\end{array}\right)\left(\begin{array}{l}
u_{\uparrow} \\
u_{\downarrow} \\
v_{\downarrow} \\
v_{\uparrow}
\end{array}\right)=0
$$

distinguishing between the two helicities \pm as

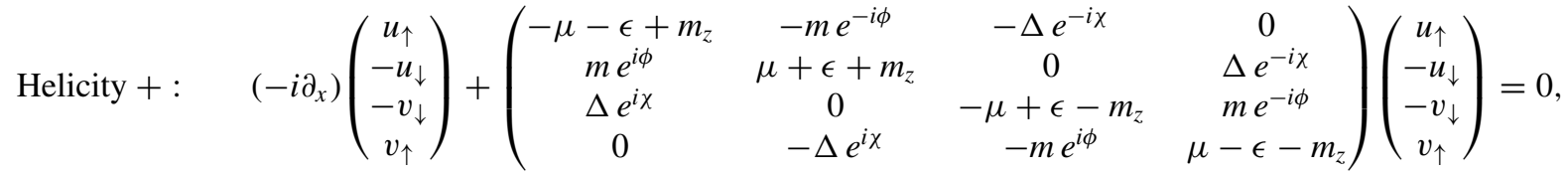

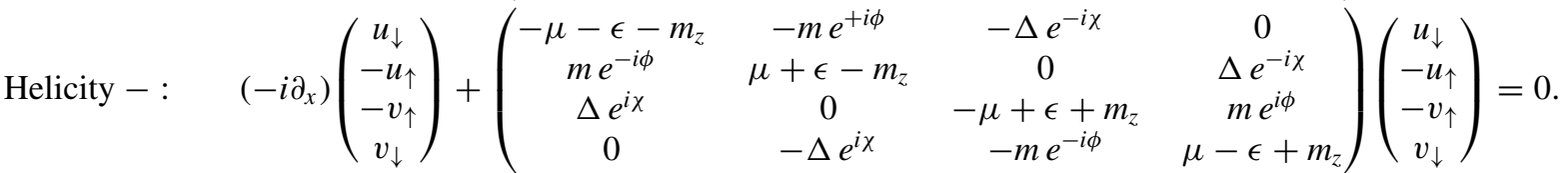


Hence, we further analyze only one helicity, say the + one, since the solutions for the other one can be easily obtained by implementing the transformation

$$
\begin{aligned}
m_{z} & \leftrightarrow-m_{z}, \\
\phi & \leftrightarrow-\phi, \\
\uparrow & \leftrightarrow \downarrow .
\end{aligned}
$$

\section{APPENDIX B: SCATTERING MATRICES}

To identify the presence of midgap bound states and to study their wave function, we employ scattering theory. To this end, we have to associate a scattering matrix with each ferromagnetic and superconducting region.

\section{Ferromagnetic gapped region}

Let us first consider a ferromagnetic gapped region, centered in $x_{0}$ with width $l$. We set $\Delta=0$ and focus only on states within the gap, i.e., with energies $|m|>|\epsilon+\mu|$. The $\mathrm{BdG}$ equation (A13) is then block-diagonal and we can focus at first on the electronic sector,

$$
\left(-i \partial_{x}\right)\left(\begin{array}{c}
u_{\uparrow} \\
-u_{\downarrow}
\end{array}\right)+\left(\begin{array}{cc}
-\mu-\epsilon+m_{z} & -m e^{-i \phi} \\
m e^{i \phi} & \mu+\epsilon+m_{z}
\end{array}\right)\left(\begin{array}{c}
u_{\uparrow} \\
-u_{\downarrow}
\end{array}\right)=0 .
$$

It admits exponential solutions at a given energy $\epsilon$, which read

$$
\begin{gathered}
u_{\uparrow}=e^{-i m_{z} x-i \phi}\left\{[-i \kappa-(\epsilon+\mu)] A_{1} e^{-x \kappa}\right. \\
\left.+[i \kappa-(\epsilon+\mu)] A_{2} e^{x \kappa}\right\}, \\
u_{\downarrow}=-m e^{-i m_{z} x}\left[A_{1} e^{-x \kappa}+A_{2} e^{x \kappa}\right],
\end{gathered}
$$

with generic complex parameters $A_{i}$ and $\kappa=$ $\sqrt{m^{2}-(\epsilon+\mu)^{2}}$. By contrast, in a gapless region with no in-plane magnetization $m=0$, the plane-wave solutions

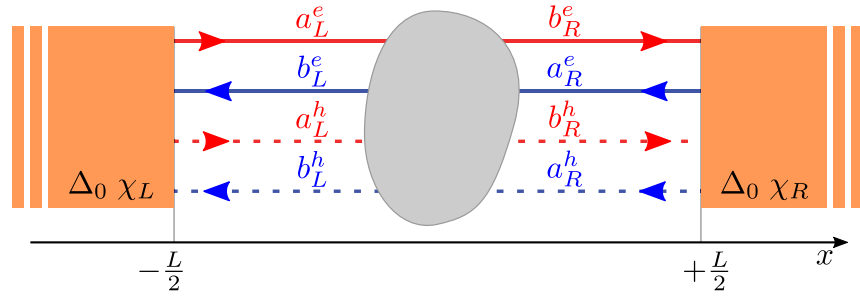

FIG. 6. A generic scattering region (gray) in between two semiinfinite superconductors (orange). Solid (dashed) lines refer to electron (hole) channels. Different colors refer to opposite spin polarizations. The scattering amplitudes are labeled in the following way: $a$ is the amplitude associated with electrons $(e)$ or holes $(h)$ incoming from the left $(L)$ or from the right $(R)$ of the gray area. Analogously, $b$ is the amplitude associated with electrons $(e)$ or holes $(h)$ outgoing to the left $(L)$ or to the right $(R)$.

read

$$
\begin{gathered}
u_{\uparrow}=C_{1} e^{i\left(\tilde{\mu}+\epsilon-\tilde{m}_{z}\right) x}, \\
u_{\downarrow}=C_{2} e^{-i\left(\tilde{\mu}+\epsilon+\tilde{m}_{z}\right) x} .
\end{gathered}
$$

For the sake of generality, we consider a nonvanishing chemical potential $(\tilde{\mu})$ and magnetization along $z\left(\tilde{m}_{z}\right)$, even outside the ferromagnetic region. By matching these solutions on the boundary of the ferromagnetic region, we can construct the scattering matrix $\mathcal{S}$ that describes it. The latter relates the incoming scattering amplitudes $\vec{a}$ with the outgoing ones $\vec{b}$ (see Fig. 6),

$$
\left(\begin{array}{c}
b_{L}^{e} \\
b_{R}^{e} \\
b_{L}^{h} \\
b_{R}^{h}
\end{array}\right)=\mathcal{S}\left(\begin{array}{c}
a_{L}^{e} \\
a_{R}^{e} \\
a_{L}^{h} \\
a_{R}^{h}
\end{array}\right) .
$$

In particular, the continuity constraint for an electronic scattering from the left reads

$$
\begin{array}{ll}
u_{\uparrow}\left(x_{0}-l / 2\right)=e^{+i\left(\tilde{\mu}+\epsilon-\tilde{m}_{z}\right)\left(x_{0}-l / 2\right)} & =e^{-i m_{z}\left(x_{0}-l / 2\right)-i \phi}\left[[-i \kappa-(\epsilon+\mu)] A_{1_{L}} e^{-\left(x_{0}-l / 2\right) \kappa}+[i \kappa-(\epsilon+\mu)] A_{2_{L}} e^{+\left(x_{0}-l / 2\right) \kappa}\right], \\
u_{\downarrow}\left(x_{0}-l / 2\right)=r_{L}^{e} e^{-i\left(\tilde{\mu}+\epsilon+\tilde{m}_{z}\right)\left(x_{0}-l / 2\right)} & =-m e^{-i m_{z}\left(x_{0}-l / 2\right)}\left[A_{1_{L}} e^{-\left(x_{0}-l / 2\right) \kappa}+A_{2_{L}} e^{+\left(x_{0}-l / 2\right) \kappa}\right], \\
u_{\uparrow}\left(x_{0}+l / 2\right)=t_{L}^{e} e^{+i\left(\tilde{\mu}+\epsilon-\tilde{m}_{z}\right)\left(x_{0}+l / 2\right)} & =e^{-i m_{z}\left(x_{0}+l / 2\right)-i \phi}\left[[-i \kappa-(\epsilon+\mu)] A_{1_{L}} e^{-\left(x_{0}+l / 2\right) \kappa}+[i \kappa-(\epsilon+\mu)] A_{2_{L}} e^{+\left(x_{0}+l / 2\right) \kappa}\right], \\
u_{\downarrow}\left(x_{0}+l / 2\right)=0 & =-m e^{-i m_{z}\left(x_{0}+l / 2\right)}\left[A_{1_{L}} e^{-\left(x_{0}+l / 2\right) \kappa}+A_{2_{L}} e^{+\left(x_{0}+l / 2\right) \kappa}\right] .
\end{array}
$$

An electronic scattering from the right is instead described by

$$
\begin{array}{ll}
u_{\uparrow}\left(x_{0}-l / 2\right)=0 & =e^{-i m_{z}\left(x_{0}-l / 2\right)-i \phi}\left[[-i \kappa-(\epsilon+\mu)] A_{1_{R}} e^{-\left(x_{0}-l / 2\right) \kappa}+[i \kappa-(\epsilon+\mu)] A_{2_{R}} e^{+\left(x_{0}-l / 2\right) \kappa}\right], \\
u_{\downarrow}\left(x_{0}-l / 2\right)=t_{R}^{e} e^{-i\left(\tilde{\mu}+\epsilon+\tilde{m}_{z}\right)\left(x_{0}-l / 2\right)} & =-m e^{-i m_{z}\left(x_{0}-l / 2\right)}\left[A_{1_{R}} e^{-\left(x_{0}-l / 2\right) \kappa}+A_{2_{R}} e^{+\left(x_{0}-l / 2\right) \kappa}\right], \\
u_{\uparrow}\left(x_{0}+l / 2\right)=r_{R}^{e} e^{+i\left(\tilde{\mu}+\epsilon-\tilde{m}_{z}\right)\left(x_{0}+l / 2\right)} & =e^{-i m_{z}\left(x_{0}+l / 2\right)-i \phi}\left[[-i \kappa-(\epsilon+\mu)] A_{1_{R}} e^{-\left(x_{0}+l / 2\right) \kappa}+[i \kappa-(\epsilon+\mu)] A_{2_{R}} e^{+\left(x_{0}+l / 2\right) \kappa}\right], \\
u_{\downarrow}\left(x_{0}+l / 2\right)=e^{-i\left(\tilde{\mu}+\epsilon+\tilde{m}_{z}\right)\left(x_{0}+l / 2\right)} & =-m e^{-i m_{z}\left(x_{0}+l / 2\right)}\left[A_{1_{R}} e^{-\left(x_{0}+l / 2\right) \kappa}+A_{2_{R}} e^{+\left(x_{0}+l / 2\right) \kappa}\right] .
\end{array}
$$

By solving these systems, we obtain

$$
\begin{gathered}
r_{L}^{e}=m e^{-i\left(l-2 x_{0}\right)(\epsilon+\tilde{\mu})} e^{+i \phi}\left(e^{2 l \kappa}-1\right) \Xi^{-1}, \\
t_{L}^{e}=2 i \kappa e^{-i l(\epsilon+\tilde{\mu})} e^{-i l\left(m_{z}-\tilde{m}_{z}\right)} e^{l \kappa} \Xi^{-1}, \\
r_{R}^{e}=m e^{-i\left(l+2 x_{0}\right)(\epsilon+\tilde{\mu})} e^{-i \phi}\left(e^{2 l \kappa}-1\right) \Xi^{-1}, \\
t_{R}^{e}=2 i \kappa e^{-i l(\epsilon+\tilde{\mu})} e^{+i l\left(m_{z}-\tilde{m}_{z}\right)} e^{l \kappa} \Xi^{-1},
\end{gathered}
$$


with

$$
\Xi=(\epsilon+\mu)\left(e^{2 l \kappa}-1\right)+i \kappa\left(1+e^{2 l \kappa}\right) .
$$

Inside the gapped region, the wave function is described by

$$
\begin{gathered}
A_{1_{L}}=-\exp \left[i \phi+2 \kappa l+\left(x_{0}-l / 2\right)\right. \\
\left.\times\left[\kappa+i\left(\epsilon+\tilde{\mu}+m_{z}-\tilde{m}_{z}\right)\right]\right] \Xi^{-1}, \\
A_{2_{L}}=-A_{1_{L}} \exp \left[-\kappa\left(2 x_{0}+l\right)\right], \\
A_{1_{R}}=-A_{2_{R}} \exp \left[-\kappa\left(2 x_{0}-l\right)\right](\epsilon+u+i \kappa)(\epsilon+u-i \kappa)^{-1}, \\
A_{2_{R}}=-(\epsilon+\mu+i \kappa) m^{-1} \exp \left[-i\left(x_{0}+l / 2\right)(\epsilon+\tilde{\mu} 16)\right. \\
\left.\left.-m_{z}+\tilde{m}_{z}\right)-x_{0} \kappa+3 l \kappa / 2\right] \Xi^{-1} .
\end{gathered}
$$

These results allow us to construct the full scattering matrix by exploiting the particle-hole symmetry of the Hamiltonian [in particular Eq. (A12)],

$$
\mathcal{S}_{\mathrm{F}}(\epsilon)=\left(\begin{array}{cccc}
r_{L}^{e}(\epsilon) & t_{R}^{e}(\epsilon) & 0 & 0 \\
t_{L}^{e}(\epsilon) & r_{R}^{e}(\epsilon) & 0 & 0 \\
0 & 0 & -r_{L}^{e}(-\epsilon)^{*} & t_{R}^{e}(-\epsilon)^{*} \\
0 & 0 & t_{L}^{e}(-\epsilon)^{*} & -r_{R}^{e}(-\epsilon)^{*}
\end{array}\right) .
$$

\section{Superconducting gapped region}

Here we consider a superconducting region, with $m=$ $m_{z}=0$, centered in $x_{0}$ and with width $l$. The BdG can be cast in a block-diagonal form and we can thus focus again only on two variables,

$$
\left(-i \partial_{x}\right)\left(\begin{array}{c}
u_{\uparrow} \\
-v_{\downarrow}
\end{array}\right)+\left(\begin{array}{cc}
-\mu-\epsilon+m_{z} & -\Delta e^{-i \chi} \\
\Delta e^{i \chi} & -\mu+\epsilon-m_{z}
\end{array}\right)\left(\begin{array}{c}
u_{\uparrow} \\
-v_{\downarrow}
\end{array}\right)=0 .
$$

Such an equation is equivalent to the one for the ferromagnet provided that the following identifications are made:

\begin{tabular}{ccc} 
Ferromagnet & & Superconductor \\
\hline$m$ & $\leftrightarrow$ & $\Delta$ \\
$\phi$ & $\leftrightarrow$ & $\chi$ \\
$m_{z}$ & $\leftrightarrow$ & $-\mu$ \\
$\mu$ & $\leftrightarrow$ & 0 \\
$\tilde{m}_{z}$ & $\leftrightarrow$ & $-\tilde{\mu}$ \\
$\tilde{\mu}$ & $\leftrightarrow$ & $-\tilde{m}_{z}$ \\
$u_{\downarrow}$ & $\leftrightarrow$ & $v_{\downarrow}$
\end{tabular}

We can thus immediately obtain the results

$$
\begin{array}{r}
r_{L}^{(1)}=\Delta e^{-i\left(l-2 x_{0}\right)\left(\epsilon-\tilde{m}_{z}\right)} e^{+i \chi}\left(e^{2 l v}-1\right) \Theta^{-1}, \\
t_{L}^{(1)}=2 i v e^{-i l\left(\epsilon-\tilde{m}_{z}\right)} e^{i l(\mu-\tilde{\mu})} e^{l v} \Theta^{-1}, \\
r_{R}^{(1)}=\Delta e^{-i\left(l+2 x_{0}\right)\left(\epsilon-\tilde{m}_{z}\right)} e^{-i \chi}\left(e^{2 l v}-1\right) \Theta^{-1}, \\
t_{R}^{(1)}=2 i v e^{-i l\left(\epsilon-\tilde{m}_{z}\right)} e^{-i l(\mu-\tilde{\mu})} e^{l v} \Theta^{-1},
\end{array}
$$

with

$$
\Theta=\epsilon\left(e^{2 l v}-1\right)+i v\left(1+e^{2 l v}\right)
$$

and $v=\sqrt{\Delta^{2}-\epsilon^{2}}$. Again, we are only considering states within the gap $|\epsilon|<|\Delta|$. The quantities with a tilde, $\tilde{\mu}$ and $\tilde{m}_{z}$, refer to chemical potential and magnetization along $z$ in the gapless regions. By exploiting particle-hole symmetry, we get the scattering matrix

$$
\mathcal{S}_{\mathrm{S}}(\epsilon)=\left(\begin{array}{cccc}
0 & t_{R}^{(1)}(-\epsilon)^{*} & -r_{L}^{(1)}(-\epsilon)^{*} & 0 \\
t_{L}^{(1)}(\epsilon) & 0 & 0 & r_{R}^{(1)}(\epsilon) \\
r_{L}^{(1)}(\epsilon) & 0 & 0 & t_{R}^{(1)}(\epsilon) \\
0 & -r_{R}^{(1)}(-\epsilon)^{*} & t_{L}^{(1)}(-\epsilon)^{*} & 0
\end{array}\right) .
$$

\section{Multiple scattering regions: Transfer matrices}

To deal with multiple scattering barriers, it is necessary to work with transfer matrices $\mathcal{T}$, which relate the left scattering amplitudes with the right ones. For example, referring to the situation depicted in Fig. 6, we would have

$$
\left(\begin{array}{l}
b_{R}^{e} \\
a_{R}^{e} \\
b_{R}^{h} \\
a_{R}^{h}
\end{array}\right)=\mathcal{T}\left(\begin{array}{l}
a_{L}^{e} \\
b_{L}^{e} \\
a_{L}^{h} \\
b_{L}^{h}
\end{array}\right) .
$$

In the presence of subsequent scattering regions 1 and 2, the combined transfer matrix is just the product of the individual transfer matrices $\mathcal{T}_{\text {res }}=\mathcal{T}_{2} \mathcal{T}_{1}$. Transfer matrices are univocally related to scattering matrices.

\section{Semi-infinite superconductors}

We next discuss the properties of semi-infinite superconductors, i.e., with a width $l \rightarrow \infty$, as sketched in Fig. 6. Let us focus at first on the left one, with pairing potential $\Delta_{0}$, phase $\chi_{L}$, and spatial extension $-\infty<x<-L / 2$. According to Eqs. (B20)-(B23), particles impinging from the right are completely Andreev-reflected back since $t_{R}^{(1)} \rightarrow 0$ and $\left|r_{R}^{(1)}\right| \rightarrow 1$. In particular, for the + helicity, one has

$$
\begin{aligned}
u_{\uparrow} & =r_{R}^{(1)}(\epsilon) v_{\downarrow}=e^{i L\left(\epsilon-\tilde{m}_{z}\right)} e^{-i \chi_{L}} \frac{\Delta_{0}}{\epsilon+i \sqrt{\Delta_{0}^{2}-\epsilon^{2}}} v_{\downarrow} \\
= & e^{i L \epsilon} \exp \left[-i \chi_{L}-i L \tilde{m}_{z}-i \arccos \left(\frac{\epsilon}{\Delta_{0}}\right)\right] v_{\downarrow}, \quad(\mathrm{B} 27) \\
v_{\uparrow} & =-r_{R}^{(1)}(-\epsilon)^{*} u_{\downarrow} \\
& =-e^{i L \epsilon} \exp \left[+i \chi_{L}+i L \tilde{m}_{z}+i \arccos \left(\frac{-\epsilon}{\Delta_{0}}\right)\right] u_{\downarrow} .
\end{aligned}
$$

The same reasoning applies to the semi-infinite superconductor, which extends from $L / 2<x<\infty$ with superconducting phase $\chi_{R}$. Then, the perfect Andreev reflection takes the form

$$
\begin{aligned}
v_{\downarrow}= & r_{L}^{(1)}(\epsilon) u_{\uparrow} \\
= & e^{i L \epsilon} \exp \left[+i \chi_{R}-i L \tilde{m}_{z}-i \arccos \left(\frac{\epsilon}{\Delta_{0}}\right)\right] u_{\uparrow}, \quad(\mathrm{B} 29) \\
u_{\downarrow} & =-r_{L}^{(1)}(-\epsilon)^{*} v_{\uparrow} \\
& =-e^{i L \epsilon} \exp \left[-i \chi_{R}+i L \tilde{m}_{z}+i \arccos \left(\frac{-\epsilon}{\Delta_{0}}\right)\right] v_{\uparrow} .
\end{aligned}
$$

Results for the opposite helicity are simply obtained by exchanging $\uparrow \leftrightarrow \downarrow$ and by changing the signs of $\tilde{m}_{z}$. 
If the generic scattering region, depicted in gray in Fig. 6, is described by the scattering matrix $\mathcal{S}_{\text {in }}$, which relates

$$
\left(\begin{array}{c}
b_{L}^{e} \\
b_{R}^{e} \\
b_{L}^{h} \\
b_{R}^{h}
\end{array}\right)=\mathcal{S}_{\text {in }}\left(\begin{array}{c}
a_{L}^{e} \\
a_{R}^{e} \\
a_{L}^{h} \\
a_{R}^{h}
\end{array}\right)
$$

we can model the perfect Andreev reflections at $x= \pm L / 2$ with the matrix $\mathcal{S}_{\text {And }}$, which reads

$$
\left(\begin{array}{l}
a_{L}^{e} \\
a_{R}^{e} \\
a_{L}^{h} \\
a_{R}^{h}
\end{array}\right)=\mathcal{S}_{\text {And }}\left(\begin{array}{l}
b_{L}^{e} \\
b_{R}^{e} \\
b_{L}^{h} \\
b_{R}^{h}
\end{array}\right)=e^{i L \epsilon} e^{-i \arccos \left(\frac{\epsilon}{\Delta_{0}}\right)}\left(\begin{array}{cccc}
0 & 0 & e^{-i\left(\chi_{L}+L \tilde{m}_{z}\right)} & 0 \\
0 & 0 & 0 & e^{-i\left(\chi_{R}-L \tilde{m}_{z}\right)} \\
e^{i\left(\chi_{L}+L \tilde{m}_{z}\right)} & 0 & 0 & 0 \\
0 & e^{i\left(\chi_{R}-L \tilde{m}_{z}\right)} & 0 & 0
\end{array}\right)\left(\begin{array}{c}
b_{L}^{e} \\
b_{R}^{e} \\
b_{L}^{h} \\
b_{R}^{h}
\end{array}\right) .
$$

Taking into account the scattering in the inner gray region as well as the Andreev reflections at $x= \pm L / 2$, one can derive the well-known compatibility condition [38] for the existence of bound states with energy $\epsilon$,

$$
\operatorname{det}\left[1-\mathcal{S}_{\text {And }}(\epsilon) \mathcal{S}_{\text {in }}(\epsilon)\right]=0 .
$$

Note that the presence of magnetization along $z$ in the gapless region $\tilde{m}_{z} \neq 0$ is harmless since it only corresponds to a shift of the two superconducting phases.

\section{APPENDIX C: EFFECTS OF FINITE CHEMICAL POTENTIAL AND/OR PERPENDICULAR MAGNETIZATION}

For the sake of simplicity, in the main text we focus only on configurations with vanishing chemical potential and zero magnetization along $z$. Importantly, we argue that their presence does not qualitatively modify the zero-energy physics of the system. To this end, it is useful to understand how Eqs. (2)-(5) of the main text are modified. In the presence of nonvanishing chemical potential and/or magnetization along $z$, the finite superconductor acts as

$$
\left(\begin{array}{c}
b_{L}^{h} \\
b_{R}^{e}
\end{array}\right)=\left(\begin{array}{cc}
-i e^{i \chi} \exp \left[i \tilde{m}_{z}\left(l-2 x_{0}\right)\right] \tanh \left(\Delta l_{\mathrm{S}}\right) & \exp \left[i l\left(\tilde{m}_{z}-\mu+\tilde{\mu}\right)\right] \operatorname{sech}\left(\Delta l_{\mathrm{S}}\right) \\
\exp \left[i l\left(\tilde{m}_{z}+\mu-\tilde{\mu}\right)\right] \operatorname{sech}\left(\Delta l_{\mathrm{S}}\right) & -i e^{-i \chi} \exp \left[i \tilde{m}_{z}\left(l+2 x_{0}\right)\right] \tanh \left(\Delta l_{\mathrm{S}}\right)
\end{array}\right)\left(\begin{array}{c}
a_{L}^{e} \\
a_{R}^{h}
\end{array}\right) .
$$

The perfect Andreev reflection at $x=-L / 2$ relates

$$
a_{L}^{e}=-i e^{-i \chi_{L}-L \tilde{m}_{z}} b_{L}^{h}
$$

The combined effect of the two superconductors leads to the relation $b_{R}^{e}=-i e^{-i \tilde{\chi}_{\text {eff }}} a_{R}^{h}$, with

$$
\begin{aligned}
\tilde{\chi}_{\mathrm{eff}}\left(\chi_{L}, \chi, \Delta l_{\mathrm{S}}\right) & =\chi-\arg \left[\frac{\exp \left[i \chi+i 2 l_{\mathrm{S}} \tilde{m}_{z}\right]+\exp \left[i \chi_{L}+i \tilde{m}_{z}\left(L+l_{\mathrm{S}}+2 x_{0}\right)\right] \tanh \left(\Delta l_{\mathrm{S}}\right)}{\exp \left[i \chi_{L}+i \tilde{m}_{z} L\right]+\exp \left[i \chi+i \tilde{m}_{z}\left(l_{\mathrm{S}}-2 x_{0}\right)\right] \tanh \left(\Delta l_{\mathrm{S}}\right)}\right] \\
& =\chi-\left(l_{\mathrm{S}}+2 x_{0}\right) \tilde{m}_{z}-\arg \left[\frac{\exp \left[i \chi+i \tilde{m}_{z}\left(l_{\mathrm{S}}-2 x_{0}-L\right)\right]+\exp \left[i \chi_{L}\right] \tanh \left(\Delta l_{\mathrm{S}}\right)}{\exp \left[i \chi_{L}\right]+\exp \left[i \chi+i \tilde{m}_{z}\left(l_{\mathrm{S}}-2 x_{0}-L\right)\right] \tanh \left(\Delta l_{\mathrm{S}}\right)}\right] .
\end{aligned}
$$

Using the known result for the SFS geometry [38], we conclude that the system hosts zero-energy modes whenever

$$
\chi_{R}=\tilde{\chi}_{\mathrm{eff}}+L \tilde{m}_{z}+2 l_{\mathrm{F}}\left(m_{z}-\tilde{m}_{z}\right)+\pi .
$$

The validity of this relation is nicely verified in Fig. 7 where we plot the energy splitting $\epsilon_{\text {Maj }}$ as a function of the phase differences $\chi_{R}-\chi_{L}$ and $\chi-\chi_{L}$ in the presence of finite chemical potentials and magnetizations along $z$. As for the localization of the Majoranas, we find

$$
\begin{aligned}
\rho=\left|\frac{b_{e}}{a_{e}}\right|^{2}= & \cosh \left(2 \Delta l_{\mathrm{S}}\right)+\sinh \left(2 \Delta l_{\mathrm{S}}\right) \\
& \times \cos \left[\chi_{L}-\chi-\tilde{m}_{z}\left(l_{\mathrm{S}}-L-2 x_{0}\right)\right] .
\end{aligned}
$$

We observe that both the localization of Majoranas and the condition to have zero-energy modes depends on the parameter $\chi-\chi_{L}+\tilde{m}_{z}\left(l_{\mathrm{S}}-L-2 x_{0}\right)$.

We can therefore conclude that the presence of finite chemical potentials and/or magnetizations along the $z$ axis does not significantly affect the behavior of the zero-energy MBS. Indeed, the latter have proved to be insensitive to variations of the chemical potential, while the presence of nonvanishing magnetization along $z$, both in the ferromagnetic and gapless regions, merely corresponds to shifts in the superconducting phases. This particular behavior, which is related to the topological origin of the MBS in our system [38], justifies that we can safely consider only the $\tilde{m}_{z}=\tilde{\mu}=\mu=0$ case in the main text. 
(a)

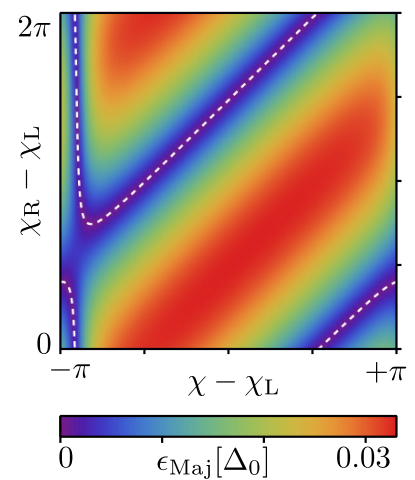

(b)

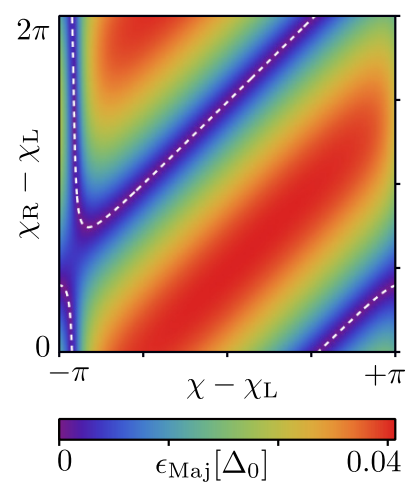

FIG. 7. Energy splitting $\epsilon_{\text {Maj }}$ (units $\Delta_{0}$ ) of the two Majoranas as a function of the superconducting phase differences. The white dashed line corresponds to the condition in Eq. (C4) and nicely highlights the presence of zero-energy MBSs. In the left panel, the ferromagnet features a nonvanishing perpendicular magnetization $m_{z}=0.2 \Delta_{0}$ while all the gapless regions feature $\tilde{m}_{z}=0.15 \Delta_{0}$. In the right panel, we add nonvanishing chemical potentials: $\mu_{\mathrm{S}}=4 \Delta_{0}, \mu_{\mathrm{F}}=0.5 \Delta_{0}$, $\tilde{\mu}=0.3 \Delta_{0}$ within the superconductor, the ferromagnet, and the gapless regions, respectively. The behavior of the system at zero energy is not affected by the chemical potential. As for the remaining parameters, in analogy with Fig. 2(a) of the main text, we choose $\Delta=m=\Delta_{0}$ and $l_{\mathrm{S}}=l_{\mathrm{F}}=\xi=\Delta_{0}^{-1}, L=7 \xi, x_{\mathrm{F}}=-x_{\mathrm{S}}=1.5 \xi$.

\section{APPENDIX D: THE QUANTUM POINT CONTACT}

Let us now consider a QPC between two helical edges with opposite helicity. We choose the the upper one to have "+" helicity, i.e., right-mover electrons have spin-up, and the lower one to have "-" helicity. At first, we focus on two gapless edges, without considering ferromagnets or superconductors. The free Hamiltonian simply reads

$$
H_{0}=\sum_{\sigma=\uparrow, \downarrow} \sum_{r=R, L} \int d x \vartheta_{r} \psi_{r \sigma}^{\dagger}(x)\left(-i \partial_{x}\right) \psi_{r \sigma}(x)
$$

with $\vartheta_{R / L}= \pm 1$. A QPC located at $x=\bar{x}$ is described by the Hamiltonian

$$
\begin{aligned}
H_{\mathrm{QPC}}= & \sum_{\sigma=\uparrow, \downarrow}\left(2 \lambda_{\mathrm{sp}} \psi_{R \sigma}^{\dagger}(\bar{x}) \psi_{L \sigma}(\bar{x})\right) \\
& +\sum_{r=R, L}\left(2 \vartheta_{r} \lambda_{\mathrm{sf}} \psi_{r \uparrow}^{\dagger}(\bar{x}) \psi_{r \downarrow}(\bar{x})\right)+\text { H.c. }
\end{aligned}
$$

where $\lambda_{\mathrm{sp}}\left(\lambda_{\mathrm{sf}}\right)$ is the spin-preserving (spin-flipping) tunneling amplitude [51]. The Heisenberg equation of motion

$$
i \partial_{t} \psi_{r \sigma}=\left[\psi_{r \sigma}, H_{\mathrm{QPC}}+H_{0}\right]
$$

gives us the following set of differential equations:

$$
\begin{aligned}
& i\left(\partial_{t}+\partial_{x}\right) \psi_{R \uparrow}=2 \delta(x-\bar{x})\left[\lambda_{\mathrm{sp}} \psi_{L \uparrow}+\lambda_{\mathrm{sf}} \psi_{R \downarrow}\right], \\
& i\left(\partial_{t}+\partial_{x}\right) \psi_{R \downarrow}=2 \delta(x-\bar{x})\left[\lambda_{\mathrm{sp}} \psi_{L \downarrow}+\lambda_{\mathrm{sf}}^{*} \psi_{R \uparrow}\right], \\
& i\left(\partial_{t}-\partial_{x}\right) \psi_{L \uparrow}=2 \delta(x-\bar{x})\left[\lambda_{\mathrm{sp}}^{*} \psi_{R \uparrow}-\lambda_{\mathrm{sf}} \psi_{L \downarrow}\right], \\
& i\left(\partial_{t}-\partial_{x}\right) \psi_{L \downarrow}=2 \delta(x-\bar{x})\left[\lambda_{\mathrm{sp}}^{*} \psi_{R \downarrow}-\lambda_{\mathrm{sf}}^{*} \psi_{L \uparrow}\right] .
\end{aligned}
$$

The plane-wave ansatz allows us to solve these equations of motions (by integrating them over an infinitesimal interval around $x=\bar{x})$. The incoming $\left(\alpha_{r \sigma}\right)$ and outgoing $\left(\beta_{r \sigma}\right)$ amplitudes satisfy the following set of equations:

$$
\begin{aligned}
i\left(\beta_{R \uparrow}-\alpha_{R \uparrow}\right)= & \lambda_{\mathrm{sp}}\left(\alpha_{L \uparrow}+\beta_{L \uparrow}\right) \exp (-2 i \epsilon \bar{x}) \\
& +\lambda_{\mathrm{sf}}\left(\alpha_{R \downarrow}+\beta_{R \downarrow}\right), \\
i\left(\beta_{R \downarrow}-\alpha_{R \downarrow}\right)= & \lambda_{\mathrm{sp}}\left(\alpha_{L \downarrow}+\beta_{L \downarrow}\right) \exp (-2 i \epsilon \bar{x}) \\
& +\lambda_{\mathrm{sf}}^{*}\left(\alpha_{R \uparrow}+\beta_{R \uparrow}\right),
\end{aligned}
$$

$$
\begin{aligned}
& \exp (-2 i \epsilon \bar{x}) i\left(\beta_{L \uparrow}-\alpha_{L \uparrow}\right) \\
& \quad=\lambda_{\mathrm{sp}}^{*}\left(\alpha_{R \uparrow}+\beta_{R \uparrow}\right)-\lambda_{\mathrm{sf}}\left(\alpha_{L \downarrow}+\beta_{L \downarrow}\right) \exp (-2 i \epsilon \bar{x}),
\end{aligned}
$$

$$
\exp (-2 i \epsilon \bar{x}) i\left(\beta_{L \downarrow}-\alpha_{L \downarrow}\right)
$$$$
=\lambda_{\mathrm{sp}}^{*}\left(\alpha_{R \downarrow}+\beta_{R \downarrow}\right)-\lambda_{\mathrm{sf}}^{*}\left(\alpha_{L \uparrow}+\beta_{L \uparrow}\right) \exp (-2 i \epsilon \bar{x}),
$$

which can be recast as

$$
\left(\begin{array}{c}
b_{L}^{e[1]} \\
b_{R}^{e[1]} \\
b_{L}^{e[2]} \\
b_{R}^{e[2]}
\end{array}\right)=\left(\begin{array}{c}
\beta_{L \downarrow} \\
\beta_{R \uparrow} \\
\beta_{L \uparrow} \\
\beta_{R \downarrow}
\end{array}\right)=\mathcal{S}_{\mathrm{QPC}}^{(e)}\left(\begin{array}{c}
a_{L}^{e[1]} \\
a_{R}^{e[1]} \\
a_{L}^{e[2]} \\
a_{R}^{e[2]}
\end{array}\right)=\mathcal{S}_{\mathrm{QPC}}^{(e)}\left(\begin{array}{c}
\alpha_{R \uparrow} \\
\alpha_{L \downarrow} \\
\alpha_{R \downarrow} \\
\alpha_{L \uparrow}
\end{array}\right),
$$

where the scattering matrix associated with the QPC reads

$$
\mathcal{S}_{\mathrm{QPC}}^{(e)}=\left(\begin{array}{cccc}
0 & \Lambda_{p f} & -\Lambda_{p b}^{*} & -\Lambda_{f f}^{*} \\
\Lambda_{p f} & 0 & -\Lambda_{f f} & \Lambda_{p b} \\
-\Lambda_{p b}^{*} & \Lambda_{f f} & 0 & \Lambda_{p f} \\
\Lambda_{f f}^{*} & \Lambda_{p b} & \Lambda_{p f} & 0
\end{array}\right)
$$

and

$$
\begin{gathered}
\Lambda_{p b}=\exp (-2 i \epsilon \bar{x}) \frac{-2 i \lambda_{\mathrm{sp}}}{1+\left|\lambda_{\mathrm{sp}}\right|^{2}+\left|\lambda_{\mathrm{sf}}\right|^{2}}, \\
\Lambda_{f f}=\frac{2 i \lambda_{\mathrm{sf}}}{1+\left|\lambda_{\mathrm{sp}}\right|^{2}+\left|\lambda_{\mathrm{sf}}\right|^{2}}, \\
\Lambda_{p f}=\frac{1-\left|\lambda_{\mathrm{sp}}\right|^{2}-\left|\lambda_{\mathrm{sf}}\right|^{2}}{1+\left|\lambda_{\mathrm{sp}}\right|^{2}+\left|\lambda_{\mathrm{sf}}\right|^{2}} .
\end{gathered}
$$

A remark on the notation: $a_{L}^{e[1]}$ is the incoming amplitude $(a)$ from the left $(L)$ of the QPC for electrons $(e)$ on the upper edge (1); the same applies for the other amplitudes.

\section{Scattering matrix in Nambu space}

To take into account the presence of superconducting and ferromagnetic gapped regions on the edges, it is useful to express the QPC scattering matrix taking also into account the tunneling of holes. We thus introduce eight-dimensional vectors of incoming and outgoing scattering amplitudes, 
$\vec{a}_{8}$ and $\vec{b}_{8}$, respectively,

$$
\vec{a}_{8}=\left(\begin{array}{c}
a_{L}^{e[1]} \\
a_{R}^{e[1]} \\
a_{L}^{h[1]} \\
a_{R}^{h[1]} \\
a_{L}^{e[2]} \\
a_{R}^{e[2]} \\
a_{L}^{h[2]} \\
a_{R}^{h[2]}
\end{array}\right), \quad \vec{b}_{8}=\left(\begin{array}{c}
b_{L}^{e[1]} \\
b_{R}^{e[1]} \\
b_{L}^{h[1]} \\
b_{R}^{h[1]} \\
b_{L}^{e[2]} \\
b_{R}^{e[2]} \\
b_{L}^{h[2]} \\
b_{R}^{h[2]}
\end{array}\right)
$$

By exploiting the particle-hole symmetry of the system, we can obtain the expression for the eight-dimensional QPC scattering matrix,

$$
\mathcal{S}_{\mathrm{QPC}}=\left(\begin{array}{cccccccc}
0 & \Lambda_{p f} & & & -\Lambda_{p b}^{*}(\epsilon) & -\Lambda_{f f}^{*} & & \\
\Lambda_{p f} & 0 & & & -\Lambda_{f f} & \Lambda_{p b}(\epsilon) & & \\
& & 0 & \Lambda_{p f} & & & \Lambda_{p b}(-\epsilon) & -\Lambda_{f f} \\
& & \Lambda_{p f} & 0 & & & -\Lambda_{f f}^{*} & -\Lambda_{p b}^{*}(-\epsilon) \\
-\Lambda_{p b}^{*}(\epsilon) & \Lambda_{f f} & & & 0 & \Lambda_{p f} & & \\
\Lambda_{f f}^{*} & \Lambda_{p b}(\epsilon) & & & \Lambda_{p f} & 0 & & \\
& & \Lambda_{p b}(-\epsilon) & \Lambda_{f f}^{*} & & & 0 & \Lambda_{p f} \\
& & \Lambda_{f f} & -\Lambda_{p b}^{*}(-\epsilon) & & & \Lambda_{p f} & 0
\end{array}\right),
$$

which relates $\vec{b}_{8}=\mathcal{S}_{\mathrm{QPC}} \vec{a}_{8}$. Note that the eight-dimensional scattering matrix associated with regions where no interedge processes are present is block-diagonal.

\section{Coupling MBS with a QPC}

We now address in more detail the effect of electron tunneling at the QPC. While it definitely induces some coupling between the two edges, it is not obvious that it can lead to the hybridization of two zero-energy MBSs located on different edges. Indeed, given two generic Majorana operators $\gamma$ and $\eta$, they can only be coupled by an imaginary hopping amplitude $H_{\text {hop }}=i \eta \gamma$. As a result, one has to carefully implement phase differences between the two edges.

To develop some intuition, it is useful to focus on the simple example depicted in Fig. 8(a). We focus only on the leftmost gapless regions of the two edges, assuming that they host zero-energy MBSs. We study the effect of a weak spin-preserving tunneling of electrons (holes) with spin up (down). The existence of MBSs at zero energy requires

$$
\left(\begin{array}{c}
a_{L}^{e[1]} \\
a_{R}^{h[1]} \\
a_{R}^{e[2]} \\
a_{L}^{h[2]}
\end{array}\right)=-i\left(\begin{array}{cccc}
e^{-i \chi_{L}^{[1]}} & 0 & 0 & 0 \\
0 & e^{i \chi_{L}^{[1]}+i \pi} & 0 & 0 \\
0 & 0 & e^{i \chi_{L}^{[2]}} & 0 \\
0 & 0 & 0 & e^{-i \chi_{L}^{[2]}+i \pi}
\end{array}\right)\left(\begin{array}{c}
b_{L}^{h[1]} \\
b_{R}^{e[1]} \\
b_{R}^{h[2]} \\
b_{L}^{e[2]}
\end{array}\right) .
$$

The spin-preserving tunneling at zero energy is described by the (reduced) scattering matrix

$$
\left(\begin{array}{l}
b_{L}^{h[1]} \\
b_{R}^{e[1]} \\
b_{R}^{h[2]} \\
b_{L}^{e[2]}
\end{array}\right)=\overline{\mathcal{S}}_{\mathrm{QPC}}\left(\begin{array}{l}
a_{L}^{e[1]} \\
a_{R}^{h[1]} \\
a_{R}^{e[2]} \\
a_{L}^{h[2]}
\end{array}\right)=\left(\begin{array}{cccc}
0 & \Lambda_{\mathrm{f}} & 0 & \Lambda_{\mathrm{b}} \\
\Lambda_{\mathrm{f}} & 0 & \Lambda_{\mathrm{b}} & 0 \\
0 & -\Lambda_{\mathrm{b}}^{*} & 0 & \Lambda_{\mathrm{f}} \\
-\Lambda_{\mathrm{b}}^{*} & 0 & \Lambda_{\mathrm{f}} & 0
\end{array}\right)\left(\begin{array}{c}
a_{L}^{e[1]} \\
a_{R}^{h[1]} \\
a_{R}^{e[2]} \\
a_{L}^{h[2]}
\end{array}\right)
$$

with forward (f) and backscattering (b) entries

$$
\Lambda_{\mathrm{f}}=\frac{1-\left|\lambda_{\mathrm{sp}}\right|^{2}}{1+\left|\lambda_{\mathrm{sp}}\right|^{2}}, \quad \Lambda_{\mathrm{b}}=\frac{-2 i \lambda_{\mathrm{sp}}}{1+\left|\lambda_{\mathrm{sp}}\right|^{2}} .
$$


(a)

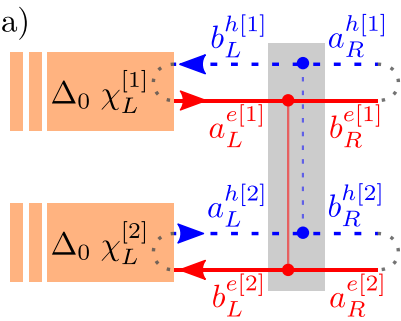

(b)

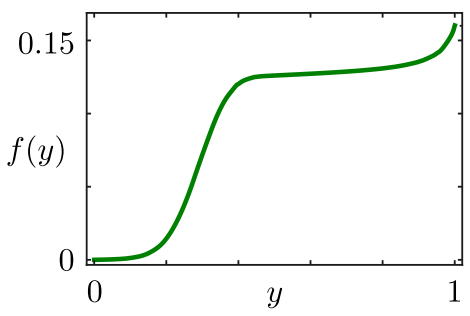

FIG. 8. (a) Sketch of spin-preserving tunnelings between the two edges. We focus only on the leftmost gapless regions of the system, depicted in Fig. 3 of the main text. Red (blue) lines refer to spin-up electron (spin-down hole) channels. (b) Plot of the correction phase $f(y)$ (in units of $\pi$ ). As in the main text, we choose $m^{[1]}=2 m^{[2]}$, $\Delta=m^{[2]}=\Delta_{0}$.

We can now inspect the effects of the QPC by considering the fate of a right-moving electron with spin up, which has just emerged from the left superconductor on the upper edge. It can follow two trajectories: it can remain on the upper edge being Andreev-reflected (i), or it can tunnel on the other edge being Andreev-reflected by the left superconductor and tunnel back (ii). Depending on the trajectory, it eventually reemerges as an electron on the upper edge with corresponding prefactors

$$
\begin{gathered}
\xi_{(i)}=\Lambda_{\mathrm{f}}\left(-i e^{i \chi_{L}^{[1]}+i \pi}\right) \Lambda_{\mathrm{f}}\left(-i e^{-i \chi_{L}^{[1]}}\right)=1-\left|\Lambda_{\mathrm{b}}\right|^{2}, \\
\xi_{(i i)}=-\Lambda_{\mathrm{p}}^{*}\left(-i e^{i \chi_{L}^{[2]}}\right) \Lambda_{\mathrm{p}}\left(-i e^{-i \chi_{L}^{[1]}}\right)=e^{i\left(\chi_{L}^{[1]}-\chi_{L}^{[2]}\right)}\left|\Lambda_{\mathrm{b}}\right|^{2} .
\end{gathered}
$$

This clearly shows that, if no phase difference between the edges is considered, $\chi_{L}^{[1]}-\chi_{L}^{[2]}=0$, spin-preserving tunneling of electrons is ineffective in coupling zero-energy MBSs.

\section{APPENDIX E: LOCAL DENSITY OF STATES}

Our goal is to compute the equilibrium zero-temperature local density of states (LDOS) on a given edge. This observable is defined as

$$
\begin{aligned}
\mathcal{A}(x, \omega)= & -\frac{1}{\pi} \operatorname{Im}\left[\operatorname{Tr} G_{e e}^{R}(x, x, \omega)\right] \\
= & -\frac{1}{\pi} \sum_{\sigma=\uparrow, \downarrow} \operatorname{Im} \int d \tau e^{i \omega \tau-0^{+} \tau} \theta(\tau) \\
& \times\left[-i\left\langle\left\{\psi_{\sigma}(x, t), \psi_{\sigma}^{\dagger}(x, t-\tau)\right\}\right\rangle_{0}\right] \\
= & \frac{1}{2 \pi} \sum_{\sigma=\uparrow, \downarrow} \int d \tau e^{i \omega \tau-0^{+}|\tau|} \\
& \times\left\langle\left\{\psi_{\sigma}(x, t), \psi_{\sigma}^{\dagger}(x, t-\tau)\right\}\right\rangle_{0},
\end{aligned}
$$

where $\langle\cdots\rangle_{0}$ is the average with respect to the ground state. By using Eqs. (A2) and (A7), the fermionic fields $\psi_{\sigma}(x)$ can be expressed as ( $\sigma=\uparrow, \downarrow= \pm)$

$$
\psi_{\sigma}(x, t)=\sum_{\epsilon} \sum_{j}\left[u_{\sigma, j}(\epsilon, x) \gamma_{\epsilon, j}(t)-\sigma v_{\sigma, j}(\epsilon, x)^{*} \gamma_{\epsilon, j}^{\dagger}(t)\right] .
$$

Given the system Hamiltonian (A6), it is straightforward to compute the ground-state averages in the nondegenerate case,

$$
\begin{aligned}
& \left\langle\left\{\psi_{\sigma}(x, t), \psi_{\sigma}^{\dagger}(x, t-\tau)\right\}\right\rangle_{0} \\
& =\sum_{\epsilon, \epsilon^{\prime} \geqslant 0}\left(u_{\sigma}(\epsilon, x) u_{\sigma}\left(\epsilon^{\prime}, x\right)^{*} e^{-i \epsilon t+i \epsilon^{\prime}(t-\tau)}\right. \\
& \left.\quad+v_{\sigma}(\epsilon, x) v_{\sigma}\left(\epsilon^{\prime}, x\right)^{*} e^{+i \epsilon t-i \epsilon^{\prime}(t-\tau)}\right)\left\langle\gamma_{\epsilon} \gamma_{\epsilon^{\prime}}^{\dagger}\right\rangle_{0} \\
& =\sum_{\epsilon \geqslant 0}\left(\left|u_{\sigma}(\epsilon, x)\right|^{2} e^{-i \epsilon \tau}+\left|v_{\sigma}(\epsilon, x)\right|^{2} e^{+i \epsilon \tau}\right) .
\end{aligned}
$$

The LDOS can be expressed as a sum of $\delta$-functions centered on the energies of the bound states. By exploiting Eq. (A12), we get

$$
\mathcal{A}(x, \omega)=\sum_{\epsilon} \sum_{\sigma}\left|u_{\sigma}(\epsilon, x)\right|^{2} \delta(\omega+\epsilon)=\sum_{\epsilon} A_{\epsilon}(x) \delta(\omega+\epsilon) .
$$

In the presence of zero-energy Majoranas, the ground state is degenerate. To deal with this subtlety, we numerically introduce a tiny perturbation in one of the phases, so that the degeneracy is lifted by a very small amount $\left(\zeta \lesssim 10^{-8} \Delta_{0}\right)$ and we computed $\left|u_{\sigma}( \pm \zeta, x)\right|^{2}$. The zero-energy LDOS plotted in the main text is then given by

$$
A_{0}(x) \simeq \sum_{\sigma}\left|u_{\sigma}(\zeta, x)\right|^{2}+\left|u_{\sigma}(-\zeta, x)\right|^{2} .
$$

\section{APPENDIX F: EXISTENCE OF ZERO-ENERGY MODES}

\section{A generic result}

We first discuss a generic property of helical systems connected to semi-infinite superconductors. In particular, we consider one semi-infinite superconductor located at $x \geqslant L / 2$, i.e., to the right of the helical system (see Fig. 6). Moreover, we make two assumptions regarding what happens to the left of the gapless helical system: (i) all the amplitudes going to the left $\left(a_{R}^{h}\right.$ and $\left.a_{R}^{e}\right)$ are completely reflected back, i.e., the system obeys some kind of open boundary conditions; (ii) the whole system is particle-hole-symmetric. Our goal is to argue that it is always possible to tune the phase $\chi_{R}$ of the right semi-infinite superconductor in order for the whole system to support zero-energy bound states.

The first assumption assures that the matrix $U(\epsilon)$,

$$
\left(\begin{array}{l}
b_{R}^{e}(\epsilon) \\
b_{R}^{h}(\epsilon)
\end{array}\right)=U(\epsilon)\left(\begin{array}{l}
a_{R}^{h}(\epsilon) \\
a_{R}^{e}(\epsilon)
\end{array}\right),
$$

is unitary. The second one, by using Eq. (A12), leads to

$$
\begin{aligned}
-\left(\begin{array}{c}
b_{R}^{h}(-\epsilon)^{*} \\
b_{R}^{e}(-\epsilon)^{*}
\end{array}\right) & =U(\epsilon)\left(\begin{array}{c}
a_{R}^{e}(-\epsilon)^{*} \\
a_{R}^{h}(-\epsilon)^{*}
\end{array}\right) \\
\Rightarrow-\left(\begin{array}{c}
b_{R}^{h}(\epsilon) \\
b_{R}^{e}(\epsilon)
\end{array}\right) & =-U(-\epsilon)^{*}\left(\begin{array}{l}
a_{R}^{e}(\epsilon) \\
a_{R}^{h}(\epsilon)
\end{array}\right) .
\end{aligned}
$$


At zero energy, the unitary matrix $U(0)$ therefore obeys

$$
\begin{aligned}
U(0) & =e^{i \varphi}\left(\begin{array}{cc}
\sin (\theta) e^{i \alpha} & \cos (\theta) e^{i \beta} \\
-\cos (\theta) e^{-i \beta} & \sin (\theta) e^{-i \alpha}
\end{array}\right) \\
& =-\left(\begin{array}{ll}
0 & 1 \\
1 & 0
\end{array}\right) U(0)^{*}\left(\begin{array}{ll}
0 & 1 \\
1 & 0
\end{array}\right),
\end{aligned}
$$

which poses constraints on the parameters $\alpha, \beta, \theta$, and $\varphi$. In particular, there are only two possibilities: either $\theta=0$ and $\varphi=0, \pi$ or $\theta=\pi / 2$ and $\varphi=\pi / 2,3 \pi / 2$. As for the right semi-infinite superconductor, at zero energy, it Andreevreflects the incoming wave function according to Eq. (B32),

$$
\left(\begin{array}{l}
a_{R}^{h} \\
a_{R}^{e}
\end{array}\right)=-i\left(\begin{array}{cc}
e^{i \chi_{R}} & 0 \\
0 & e^{-i \chi_{R}}
\end{array}\right)\left(\begin{array}{l}
b_{R}^{e} \\
b_{R}^{h}
\end{array}\right) .
$$

The whole system admits bound states at zero energy if it is possible to solve

$$
\begin{aligned}
\left(\begin{array}{l}
b_{R}^{e} \\
b_{R}^{h}
\end{array}\right)= & e^{i \varphi}\left(\begin{array}{cc}
\sin (\theta) e^{i \alpha} & \cos (\theta) e^{i \beta} \\
-\cos (\theta) e^{-i \beta} & \sin (\theta) e^{-i \alpha}
\end{array}\right) \\
& \times\left(\begin{array}{cc}
-i e^{i \chi_{R}} & 0 \\
0 & -i e^{-i \chi_{R}}
\end{array}\right)\left(\begin{array}{c}
b_{R}^{e} \\
b_{R}^{h}
\end{array}\right) \\
= & e^{i \varphi-i \frac{\pi}{2}}\left(\begin{array}{cc}
\sin (\theta) e^{i \alpha} e^{i \chi_{R}} & \cos (\theta) e^{i \beta} e^{-i \chi_{R}} \\
-\cos (\theta) e^{-i \beta} e^{i \chi_{R}} & \sin (\theta) e^{-i \alpha} e^{-i \chi_{R}}
\end{array}\right)\left(\begin{array}{l}
b_{R}^{e} \\
b_{R}^{h}
\end{array}\right),
\end{aligned}
$$

which is equivalent to requiring

$$
\begin{aligned}
& \operatorname{det}\left[1-e^{i \varphi-i \frac{\pi}{2}}\left(\begin{array}{cc}
\sin (\theta) e^{i \alpha} e^{i \chi_{R}} & \cos (\theta) e^{i \beta} e^{-i \chi_{R}} \\
-\cos (\theta) e^{-i \beta} e^{i \chi_{R}} & \sin (\theta) e^{-i \alpha} e^{-i \chi_{R}}
\end{array}\right)\right] \\
& =0 \Rightarrow \sin (\theta) \cos \left(\alpha+\chi_{\mathrm{R}}\right)-\sin (\varphi)=0 \text {. }
\end{aligned}
$$

In the $\theta=0$ case, there is therefore always a solution at zero energy regardless of the values of $\beta$ and $\chi_{R}$. In the $\theta=\pi / 2$ case, it is possible to tune $\chi_{R}=\varphi-\pi / 2-\alpha$, which results in zero-energy bound states.

\section{Zero-energy modes in the protocol discussed in the main text}

We are now in a position to comment on the tiny correction $f(y)$, which appears in the protocol that moves Majoranas from one edge to the other one. Without implementing such a correction, the protocol turns out to be qualitatively correct, but it fails to keep the MBS exactly at zero energy. This has to be expected since the protocol has been designed by exploiting the exact knowledge of the single-edge SSFS architectures supplemented with an approximate and qualitative description of the QPC.

Interestingly, it turns out that this subtlety can be straightforwardly fixed. The general result discussed above ensures that, for each value of $y$, one can force the MBS to be at zero energy just by adding a proper correction $f(y)$ to one phase, say $\chi_{R}^{[1]}$. The function $f(y)$ can be easily computed by numerically requiring the MBS to be at zero energy, and the result is plotted in Fig. 8 (right panel). Remarkably enough, it turns out to be a smooth function that features only small values $[0 \leqslant f(y) \leqslant 0.15 \pi]$ and therefore does not qualitatively affect the protocol.
[1] J. Alicea, New directions in the pursuit of Majorana fermions in solid state systems, Rep. Prog. Phys. 75, 076501 (2012).

[2] C. Beenakker, Search for Majorana fermions in superconductors, Ann. Rev. Condens. Matter Phys. 4, 113 (2013).

[3] A. Y. Kitaev, Unpaired Majorana fermions in quantum wires, Phys. Usp. 44, 131 (2001).

[4] C. Nayak, S. H. Simon, A. Stern, M. Freedman, and S. Das Sarma, Non-Abelian anyons and topological quantum computation, Rev. Mod. Phys. 80, 1083 (2008).

[5] D. Aasen, M. Hell, R. V. Mishmash, A. Higginbotham, J. Danon, M. Leijnse, T. S. Jespersen, J. A. Folk, C. M. Marcus, K. Flensberg, and J. Alicea, Milestones Toward Majorana-Based Quantum Computing, Phys. Rev. X 6, 031016 (2016).

[6] Y. Oreg, G. Refael, and F. von Oppen, Helical Liquids and Majorana Bound States in Quantum Wires, Phys. Rev. Lett. 105, 177002 (2010).

[7] R. M. Lutchyn, J. D. Sau, and S. Das Sarma, Majorana Fermions and a Topological Phase Transition in Semiconductor-Superconductor Heterostructures, Phys. Rev. Lett. 105, 077001 (2010).

[8] A. P. Higginbotham, S. M. Albrecht, G. Kiršanskas, W. Chang, F. Kuemmeth, P. Krogstrup, T. S. Jespersen, J. Nygård, K. Flensberg, and C. M. Marcus, Parity lifetime of bound states in a proximitized semiconductor nanowire, Nat. Phys. 11, 1017 (2015).

[9] V. Mourik, K. Zuo, S. M. Frolov, S. R. Plissard, E. P. A. M. Bakkers, and L. P. Kouwenhoven, Signatures of Majorana fermions in hybrid superconductor-semiconductor nanowire devices, Science 336, 1003 (2012).

[10] S. M. Albrecht, A. P. Higginbotham, M. Madsen, F. Kuemmeth, T. S. Jespersen, J. Nygård, P. Krogstrup, and C. M. Marcus, Exponential protection of zero modes in Majorana islands, Nature (London) 531, 206 (2016).

[11] M. T. Deng, S. Vaitiekenas, E. B. Hansen, J. Danon, M. Leijnse, K. Flensberg, J. Nygård, P. Krogstrup, and C. M. Marcus, Majorana bound state in a coupled quantum-dot hybrid-nanowire system, Science 354, 1557 (2016).

[12] F. Nichele, A. C. C. Drachmann, A. M. Whiticar, E. C. T O'Farrell, H. J. Suominen, A. Fornieri, T. Wang, G. C. Gardner, C. Thomas, A. T. Hatke, P. Krogstrup, M. J. Manfra, K. Flensberg, and C. M. Marcus, Scaling of Majorana Zero-Bias Conductance Peaks, Phys. Rev. Lett. 119, 136803 (2017).

[13] Ö. Gül, H. Zhang, J. D. S. Bommer, M. W. A. de Moor, D. Car, S. R. Plissard, E. P. A. M. Bakkers, A. Geresdi, K. Watanabe, T. Taniguchi, and L. P. Kouwenhoven, Ballistic Majorana nanowire devices, Nat. Nanotechnol. 13, 192 (2018). 
[14] M. T. Deng, C. L. Yu, G. Y. Huang, M. Larsson, P. Caroff, and H. Q. Xu, Anomalous zero-bias conductance peak in a Nb-InSb nanowire-Nb hybrid device, Nano Lett. 12, 6414 (2012).

[15] E. J. H. Lee, X. Jiang, R. Aguado, G. Katsaros, C. M. Lieber, and S. De Franceschi, Zero-bias Anomaly in a Nanowire Quantum Dot Coupled to Superconductors, Phys. Rev. Lett. 109, 186802 (2012).

[16] S. Nadj-Perge, I. K. Drozdov, J. Li, H. Chen, S. Jeon, J. Seo, A. H. MacDonald, B. A. Bernevig, and A. Yazdani, Observation of Majorana fermions in ferromagnetic atomic chains on a superconductor, Science 346, 602 (2014).

[17] R. Pawlak, M. Kisiel, J. Klinovaja, T. Meier, S. Kawai, T. Glatzel, D. Loss, and E. Meyer, Probing atomic structure and Majorana wave functions in mono-atomic Fe chains on superconducting Pb surface, npj Quantum Inf. 2, 16035 (2016).

[18] B. E. Feldman, M. T. Randeria, J. Li, S. Jeon, Y. Xie, Z. Wang, I. K. Drozdov, B. Andrei Bernevig, and A. Yazdani, High-resolution studies of the majorana atomic chain platform, Nat. Phys. 13, 286 (2017).

[19] M. Ruby, F. Pientka, Y. Peng, F. von Oppen, B. W. Heinrich, and K. J. Franke, End States and Subgap Structure in ProximityCoupled Chains of Magnetic Adatoms, Phys. Rev. Lett. 115, 197204 (2015).

[20] H. S. Røising, R. Ilan, T. Meng, S. H. Simon, and F. Flicker, Finite temperature effects on Majorana bound states in chiral $p$-wave superconductors, SciPost Phys. 6, 55 (2019).

[21] J.-P. Xu, M.-X. Wang, Z. L. Liu, J.-F. Ge, X. Yang, C. Liu, Z. A. Xu, D. Guan, C. L. Gao, D. Qian, Y. Liu, Q.-H. Wang, F.-C. Zhang, Q.-K. Xue, and J.-F. Jia, Experimental Detection of a Majorana Mode in the Core of a Magnetic Vortex Inside a Topological Insulator-Superconductor $\mathrm{bi}_{2} \mathrm{te}_{3} / \mathrm{nbse}_{2}$ Heterostructure, Phys. Rev. Lett. 114, 017001 (2015).

[22] C. W. J. Beenakker, P. Baireuther, Y. Herasymenko, I. Adagideli, L. Wang, and A. R. Akhmerov, Deterministic Creation and Braiding of Chiral Edge Vortices, Phys. Rev. Lett. 122, 146803 (2019).

[23] C. Malciu, L. Mazza, and C. Mora, Braiding Majorana zero modes using quantum dots, Phys. Rev. B 98, 165426 (2018).

[24] E. Prada, R. Aguado, and P. San-Jose, Measuring Majorana nonlocality and spin structure with a quantum dot, Phys. Rev. B 96, 085418 (2017).

[25] S. S. Hegde, Y. Wang, E. Huemiller, G. Yue, D. J. Van Harlingen, and S. Vishveshwara, A topological Josephson junction platform for creating, manipulating, and braiding Majorana bound states, arXiv:1907.02935.

[26] L. Fu and C. L. Kane, Superconducting Proximity Effect and Majorana Fermions at the Surface of a Topological Insulator, Phys. Rev. Lett. 100, 096407 (2008).

[27] S.-J. Choi and H. S. Sim, Non-Abelian evolution of a Majorana train in a single Josephson junction, arXiv:1808.08714 [condmat.mes-hall].

[28] S.-J. Choi and H. S. Sim, Josephson junction of finite-size superconductors on a topological insulator under a magnetic field, arXiv:1908.11403 [cond-mat.mes-hall].

[29] S. Guiducci, M. Carrega, F. Taddei, G. Biasiol, H. Courtois, F. Beltram, and S. Heun, Full electrostatic control of quantum interference in an extended trenched Josephson junction, Phys. Rev. B 99, 235419 (2019).

[30] S. Guiducci, M. Carrega, G. Biasiol, L. Sorba, F. Beltram, and S. Heun, Toward quantum Hall effect in a Joseph- son junction, Phys. Status Solidi (RRL) 13, 1800222 (2018).

[31] F. Pientka, A. Keselman, E. Berg, A. Yacoby, A. Stern, and B. I. Halperin, Topological Superconductivity in a Planar Josephson Junction, Phys. Rev. X 7, 021032 (2017).

[32] A. Fornieri, A. M. Whiticar, F. Setiawan, E. Portolés, A. C. C. Drachmann, A. Keselman, S. Gronin, C. Thomas, T. Wang, R. Kallaher, G. C. Gardner, E. Berg, M. J. Manfra, A. Stern, C. M. Marcus, and F. Nichele, Evidence of topological superconductivity in planar Josephson junctions, Nature (London) 569, 89 (2019).

[33] M. König, S. Wiedmann, C. Brüne, A. Roth, H. Buhmann, L. W. Molenkamp, X.-L. Qi, and S.-C. Zhang, Quantum spin Hall insulator state in HgTe quantum wells, Science 318, 766 (2007).

[34] C. Brüne, A. Roth, H. Buhmann, E. M. Hankiewicz, L. W. Molenkamp, J. Maciejko, X.-L. Qi, and S.-C. Zhang, Spin polarization of the quantum spin Hall edge states, Nat. Phys. 8, 485 (2012).

[35] I. Knez, R.-R. Du, and G. Sullivan, Evidence for Helical Edge Modes in Inverted InAs/GaSb Quantum Wells, Phys. Rev. Lett. 107, 136603 (2011).

[36] B. A. Bernevig, T. L. Hughes, and S.-C. Zhang, Quantum spin Hall effect and topological phase transition in $\mathrm{HgTe}$ quantum wells, Science 314, 1757 (2006).

[37] L. Fu and C. L. Kane, Josephson current and noise at a superconductor/quantum-spin-Hall-insulator/superconductor junction, Phys. Rev. B 79, 161408(R) (2009).

[38] F. Crépin, B. Trauzettel, and F. Dolcini, Signatures of Majorana bound states in transport properties of hybrid structures based on helical liquids, Phys. Rev. B 89, 205115 (2014).

[39] F. Crépin, P. Burset, and B. Trauzettel, Odd-frequency triplet superconductivity at the helical edge of a topological insulator, Phys. Rev. B 92, 100507(R) (2015).

[40] F. Keidel, P. Burset, and B. Trauzettel, Tunable hybridization of Majorana bound states at the quantum spin Hall edge, Phys. Rev. B 97, 075408 (2018).

[41] J. Li, W. Pan, B. A. Bernevig, and R. M. Lutchyn, Detection of Majorana Kramers Pairs Using a Quantum Point Contact, Phys. Rev. Lett. 117, 046804 (2016).

[42] J. I. Väyrynen, G. Rastelli, W. Belzig, and L. I. Glazman, Microwave signatures of Majorana states in a topological Josephson junction, Phys. Rev. B 92, 134508 (2015).

[43] U. Borla, D. Kuzmanovski, and A. M. Black-Schaffer, Tuning Majorana zero modes with temperature in $\pi$-phase Josephson junctions, Phys. Rev. B 97, 014507 (2018).

[44] E. Bocquillon, R. S. Deacon, J. Wiedenmann, P. Leubner, T. M. Klapwijk, C. Brüne, K. Ishibashi, H. Buhmann, and L. W. Molenkamp, Gapless Andreev bound states in the quantum spin Hall insulator HgTe, Nat. Nanotechnol. 12, 137 (2016).

[45] J. Strunz, J. Wiedenmann, C. Fleckenstein, L. Lunczer, W. Beugeling, V. L. Müller, P. Shekhar, N. Traverso Ziani, S. Shamim, J. Kleinlein, H. Buhmann, B. Trauzettel, and L. W. Molenkamp, Interacting topological edge channels, Nat. Phys. (2019), doi: 10.1038/s41567-019-0692-4.

[46] S. Park and P. Recher, Detecting the Exchange Phase of Majorana Bound States in a Corbino Geometry Topological Josephson Junction, Phys. Rev. Lett. 115, 246403 (2015).

[47] S. Mi, D. I. Pikulin, M. Wimmer, and C. W. J. Beenakker, Proposal for the detection and braiding of Majorana fermions 
in a quantum spin Hall insulator, Phys. Rev. B 87, 241405(R) (2013).

[48] C. Chamon, R. Jackiw, Y. Nishida, S.-Y. Pi, and L. Santos, Quantizing Majorana fermions in a superconductor, Phys. Rev. B 81, 224515 (2010).

[49] In the limiting case $\left(\Delta l_{\mathrm{S}}\right) \rightarrow \infty$, the system effectively consist of two uncoupled junctions, SS and SFS, which can both accommodate a couple of zero-energy Majoranas. When the central superconductor has finite strength $\left(\Delta l_{\mathrm{S}}\right)$, there is a finite coupling between the junctions proportional to $\exp \left[-\Delta l_{\mathrm{S}}\right]$ and the four Majoranas hybridize. As a result, only a single pair of Majoranas can be at zero energy while the other pair will always feature a minimal hybridization energy proportional to $\exp \left[-\Delta l_{\mathrm{S}}\right]$.

[50] J. D. Pillet, V. Benzoni, J. Griesmar, J. L. Smirr, and Ç. Ö. Girit, Non-local Josephson effect in Andreev molecules, Nano Lett. 19, 7138 (2019).

[51] D. Ferraro, C. Wahl, J. Rech, T. Jonckheere, and T. Martin, Electronic Hong-Ou-Mandel interferometry in twodimensional topological insulators, Phys. Rev. B 89, 075407 (2014).

[52] N. Pankratova, H. Lee, R. Kuzmin, M. Vavilov, K. Wickramasinghe, W. Mayer, J. Yuan, J. Shabani, and V. E. Manucharyan, The multi-terminal Josephson effect, arXiv:1812.06017 [cond-mat.supr-con].

[53] R.-P. Riwar, M. Houzet, J. S. Meyer, and Y. V. Nazarov, Multi-terminal Josephson junctions as topological matter, Nat. Commun. 7, 11167 (2016).

[54] H. Ren, F. Pientka, S. Hart, A. T. Pierce, M. Kosowsky, L. Lunczer, R. Schlereth, B. Scharf, E. M. Hankiewicz, L. W. Molenkamp, B. I. Halperin, and A. Yacoby, Topological superconductivity in a phase-controlled Josephson junction, Nature (London) 569, 93 (2019).
[55] B. van Heck, A. R. Akhmerov, F. Hassler, M. Burrello, and C. W. J. Beenakker, Coulomb-assisted braiding of Majorana fermions in a Josephson junction array, New J. Phys. 14, 035019 (2012).

[56] T. Zhou, M. C. Dartiailh, W. Mayer, J. E. Han, A. MatosAbiague, J. Shabani, and I. Zutic, Phase control of Majorana bound states in a topological X junction, arXiv:1909.05386 [cond-mat.mes-hall].

[57] J. Wiedenmann, E. Bocquillon, R. S. Deacon, S. Hartinger, O. Herrmann, T. M. Klapwijk, L. Maier, C. Ames, C. Brüne, C. Gould, A. Oiwa, K. Ishibashi, S. Tarucha, H. Buhmann, and L. W. Molenkamp, $4 \pi$-periodic Josephson supercurrent in HgTe-based topological Josephson junctions, Nat. Commun. 7 (2016).

[58] E. Bocquillon, J. Wiedenmann, R. S. Deacon, T. M. Klapwijk, H. Buhmann, and L. W. Molenkamp, Microwave studies of the fractional Josephson effect in HgTe-based Josephson junctions, in Topological Matter, edited by D. Bercioux, J. Cayssol, M. Vergniory, and C. M. Reyes, Springer Series in Solid-State Sciences, Vol. 190 (Springer, Cham, 2018), pp. 115-148.

[59] J. Sánchez-Barriga, J. Braun, J. Minár, I. Di Marco, A. Varykhalov, O. Rader, V. Boni, V. Bellini, F. Manghi, H. Ebert, M. I. Katsnelson, A. I. Lichtenstein, O. Eriksson, W. Eberhardt, H. A. Dürr, and J. Fink, Effects of spin-dependent quasiparticle renormalization in $\mathrm{Fe}, \mathrm{Co}$, and Ni photoemission spectra: An experimental and theoretical study, Phys. Rev. B 85, 205109 (2012).

[60] J. C. Budich, S. Walter, and B. Trauzettel, Failure of protection of Majorana based qubits against decoherence, Phys. Rev. B 85, 121405(R) (2012).

[61] D. Rainis and D. Loss, Majorana qubit decoherence by quasiparticle poisoning, Phys. Rev. B 85, 174533 (2012). 